\title{
Microphysics of cosmic ray driven plasma instabilities
}

\author{
A.M. Bykov • A. Brandenburg • \\ M.A. Malkov • S.M. Osipov
}

Received: date / Accepted: date

\begin{abstract}
Energetic nonthermal particles (cosmic rays, CRs) are accelerated in supernova remnants, relativistic jets and other astrophysical objects. The CR energy density is typically comparable with that of the thermal components and magnetic fields. In this review we discuss mechanisms of magnetic field amplification due to instabilities induced by CRs. We derive CR kinetic and magnetohydrodynamic equations that govern cosmic plasma systems comprising the thermal background plasma, comic rays and fluctuating magnetic fields to study CR-driven instabilities. Both resonant and non-resonant instabilities are reviewed, including the Bell short-wavelength instability, and the firehose instability. Special attention is paid to the longwavelength instabilities driven by the CR current and pressure gradient. The helicity production by the CR current-driven instabilities is discussed in connection with the dynamo mechanisms of cosmic magnetic field amplification.
\end{abstract}

Keywords magnetic fields $\cdot$ cosmic rays $\cdot$ collisionless shocks $\cdot$ supernova remnants

A.M. Bykov
A.F. Ioffe Institute for Physics and Technology, 194021, St.Petersburg, Russia
and St.Petersburg State Politechnical University, E-mail: byk@astro.ioffe.ru

A. Brandenburg

Nordita, Royal Institute of Technology and Stockholm University, Roslagstullsbacken 23, 10691 Stockholm, Sweden; and Department of Astronomy, Stockholm University, SE 10691 Stockholm, Sweden, E-mail: brandenb@nordita.org

M.A. Malkov

University of California, San Diego, La Jolla, California 92093, USA E-mail: mmalkov@ucsd.edu

S.M. Osipov

A.F. Ioffe Institute for Physics and Technology, 194021, St.Petersburg, Russia Email: osm2004@mail.ru 


\section{Introduction}

Acceleration of cosmic rays (CRs) in the Galaxy by the first order Fermi mechanism is believed to be very efficient. Most of the theoretical studies of shock acceleration agree on its potential to convert, under favorable conditions, $50 \%$ or more of shock mechanical energy into the CR energy. Observational estimates of the supernova remnant (SNR) shock power require, on the average, a 15-30\% conversion efficiency to maintain the observed CR energy against losses from the Galaxy (see, e.g., Berezinskii et al. 1990, Drury et al. 1989). However, this acceleration mechanism is fast enough only if it is selfsustained; accelerated particles must be scattered across the shock at an enhanced rate (to gain energy rapidly) by magnetic irregularities amplified by the particles themselves. Relying on the background magnetic irregularities (interstellar medium [ISM] turbulence) would result only in a very slow acceleration.

Fortunately, freshly accelerated CRs indeed comprise enough free energy to drive plasma instabilities thus bootstrapping their own acceleration (see, e.g., Zweibel 1979). While they are accumulated in a relatively thin layer near a shock front, their pressure gradient is built up. Furthermore, they stream through the inflowing plasma so that their pitch-angle distribution is anisotropic. They also provide an electric current and induce a return current in the upstream plasma.

Instabilities driven by the above sources of free energy may loosely be categorized as follows. First, an ion-cyclotron type, resonant instability (driven by the CR anisotropy) amplifies Alfven and magnetosonic waves, with no major changes to their dispersive properties and the macroscopic state of the medium near the shock. However, the amplified waves make the CR pressure and current to build-up rapidly through an enhanced CR scattering and energy gain. Second, there is a non-resonant firehose type instability driven by the CR pressure anisotropy. In contrast to the resonant instability, the firehose instability changes the Alfven wave dispersive properties by making the growing mode aperiodic. So does the current driven non-resonant instability. The renewed interest to this instability has been sparked by Bell (2004), who revealed its potential to strongly amplify the background magnetic field. Indeed, a formal analytic solution in which the instability driver is balanced by the nonlinearity indicates that the instability saturates only at very high amplitudes, $\delta B \gg B_{0}$ (see e.g. Bell and Lucek 2001, Bell 2005, Marcowith et al. 2006, Caprioli et al. 2008, Vladimirov et al. 2009, Malkov et al. 2012b). Finally, the CR pressure gradient in the shock precursor drives acoustic perturbations. All these instabilities should be treated on a unified basis, as they are driven by the anisotropic inhomogeneous CR plasma component near a shock front. An attempt of such treatment is presented below. However a complete nonlinear study of these phenomena is a formidable task, yet to be accomplished.

While the above instabilities, clearly associated with collisionless shocks, will be central to the present review, CRs are also known to drive instabilities crucial to their confinement regardless of the way they are accelerated. For example, a sufficiently dense CR cloud released into the ISM will drive Alfven 
waves which, in turn, will scatter the CRs, thus delaying their escape (see e.g. Ptuskin et al. 2008, Ohira et al. 2011, Malkov et al. 2012a, Yan et al. 2012). Moving further out to the CR confinement in the galaxy, the so-called Parker instability is known to be important, in addition to the Alfven wave self-generation by escaping CRs.

The diffusive shock acceleration (DSA) mechanism is based on repeated shock crossings with $\mathrm{a} \sim u_{\mathrm{s}} / c$ particle energy gain per cycle (see Krymskii 1977, Bell 1978, Blandford and Eichler 1987, Berezhko and Krymskii 1988, Jones and Ellison 1991). While doing so, particles diffusively escape from the shock up to a distance $L_{p} \sim \kappa(p) / u_{s}$. Here $\kappa$ is the momentum dependent diffusion coefficient and $u_{\mathrm{s}}$ is the shock velocity. One should expect then an extended $\left(\sim L_{p}\right)$ shock precursor populated by accelerated protons and electrons so that synchrotron radiating electrons may make it visible. Highresolution $X$-ray observations have revealed thin X-ray synchrotron filaments and fast evolving clumps in synchrotron emitting supernova shells. The filaments are much thinner than $L_{p}$ because the TeV regime electrons are confined in a narrow layer around the shock. Most likely they are limited by fast synchrotron cooling due to the X-ray emission in a highly amplified magnetic field (see for review Cassam-Chenaï et al. 2007, Reynolds 2008, Vink 2012, Helder et al. 2012). The synchrotron emission clumps with a year time scale variability observed with Chandra observatory by Uchivama et al. (2007) can be associated with strong intermittency of the amplified magnetic fields (Bykov et al. 2008). Moreover, a quasi-regular set of strips of synchrotron emission resolved with Chandra in Tycho's SNR by Eriksen et al. (2011) potentially can be used to study a specific angular dependence and the spectral properties of nonlinear mechanisms of magnetic field amplification by CRdriven instabilities (Bykov et al. 2011a).

According to the widely accepted view, the particle diffusion coefficient $\kappa$ should be close to the Bohm value, $\kappa \sim c r_{g}(p) / 3$, which requires strong magnetic fluctuations $\delta B_{k} \sim B_{0}$ at the resonant scale $k \sim 1 / r_{g}(p)$. The high level of fluctuations is achieved through one of the instabilities driven by accelerated particles. A number of CR driven instabilities have been suggested to generate magnetic field fluctuations. The first one is the well known ion cyclotron resonant instability of a slightly anisotropic (in pitch angle) CR distribution (see e.g. Sagdeev and Shafranov 1961, Zweibel 1979, Schlickeiser 2002, Amato 2011). The free energy source of this instability is potentially sufficient to generate magnetic field fluctuations needed to scatter CRs ahead of the shock (see e.g. Bell 1978, McKenzie and Voelk 1982).

$$
\left(\delta B / B_{0}\right)^{2} \sim M_{A} P^{c r} / \rho u_{\mathrm{s}}^{2} .
$$

where $M_{A} \gg 1$ is the Alfvenic Mach number, $P^{c r}$ is the CR pressure, $\rho$ is the gas density and $u_{\mathrm{S}}$ is the shock velocity. However, the actual turbulence level was shown to remain moderate, $\delta B \sim B_{0}$ as this is a resonant kinetic instability that is usually suppressed by a quasilinear isotropisation or particle trapping effects easily (see e.g. McKenzie and Voelk 1982, Achterberg and Blandford 1986, Zweibel 2003).

The second instability, is a nonresonant instability driven by the CR current. The advantage of this instability seems to be twofold. First, it cannot 
be stabilized by the quasilinear deformation of the $\mathrm{CR}$ distribution function since in the upstream plasma frame the driving CR current persists, once the $\mathrm{CR}$ cloud is at rest in the shock frame. Second, it generates a broad spectrum of waves, and the longest ones were claimed to be stabilized only at the level $\delta B \gg B_{0}$, due to the lack of efficient stabilization mechanism at such scales (see e.g. Bell 2004). Within the context of the CR acceleration, this instability was studied by Achterberg (1983) (see also Shapiro et al. 1998), but the fast regime of the nonresonant instability was found by Bell and Lucek (2001) and Bell (2004), and therefore the instability is often referred to as Bell's instability. Bell (2004) pointed out that in the instability is driven by a fixed CR return current through the Ampere force $\mathbf{j}_{c r} \times \mathbf{B}$. It should be noted, however, that the dissipation of the return current due to the anomalous resistivity still needs to be addressed. The effect of a finite plasma temperature on the instability was studied by Zweibel and Everett (2010). Actually, as we will show below, both the resonant and the Bell instabilities are interconnected, they are driven by the CR drift relative the background plasma. Moreover, in the case of the modes propagating along the mean magnetic fields the two instabilities are simultaneously influencing the same modes. The dispersion relations of the modes are strongly influenced by the presence of the CR current are markedly different from the standard MHD modes. The dispersion relations of the modes strongly influenced by the presence of the CR current are markedly different from the standard MHD modes. The dispersion relation in the longwavelength regime (where the mode wavelengths are larger than the bulk CR gyroradii) can be also strongly modified by the ponderomotive forces induced by Bell's turbulence. The longwavelength instability has two regimes (Bykov et al. 2011c, 2012). The first regime is prominent in the intermediate range where the mode wavelength is above the CR gyroradii but below the CR mean free path. It is discussed in 4.4 and is associated with a dynamo type instability driven by the nonzero helicity, which is, in turn, produced by the short scale CR-driven turbulence. The intermediate wavenumber range is rather narrow in the case of the Bohm-type CR diffusion. The modes with wavelengths larger than the $\mathrm{CR}$ mean free path are subject of non-resonant long-wavelength instability caused by the ponderomotive force acting on the background plasma that is induced by Bell's turbulence. We discuss the long wavelength instability below in 4.5 .

The third instability is an acoustic instability (also known as Drury's instability) driven by the pressure gradient of accelerated CRs upstream (Dorfi and Drury 1985, Drury and Falle 1986, Drury and Downes 2012, Schure et al. 2012). The pressure gradient is clearly a viable source of free energy for the instability. So, among the macroscopic quantities varying across a strong shock, the pressure jump is the most pronounced one in that it does not saturate with the Mach number, unlike the density or velocity jumps.

The acoustic instability has received somewhat less attention than the first two. Moreover, in many numerical studies of the CR shock acceleration, special care is taken to suppress it. The suppression is achieved by using the fact that a change of stability occurs at that point in the flow where $\partial \ln \kappa / \partial \ln \rho \simeq-1$ (for both stable and unstable wave propagation directions, of course, if such point exists at all). Here $\rho$ is the gas density. Namely, 
one requires this condition to hold identically all across the shock precursor, i.e., where the CR pressure gradient $\nabla P^{c r} \neq 0$. Not only is this requirement difficult to justify physically, but, more importantly, an artificial suppression of the instability eliminates its genuine macroscopic and microscopic consequences, as briefly discussed below.

Among the macroscopic consequences an important one is the vorticity generation through the baroclinic effect (missalignment of the density and pressure gradients $\nabla \rho \times \nabla P \neq 0$, e.g. Ryu et al. (1993), Kulsrud et al. (1997)) . Here $\nabla P$ may be associated with a quasi-constant macroscopic CRgas pressure gradient $\nabla P^{c r}$, generally directed along the shock normal. Variations of $\nabla \rho$ are locally decoupled from $P^{c r}$, unlike in the situation in a gas with a conventional equation of state where $P=P(\rho)$ and where the baroclinic term vanishes. The vorticity generation obviously results (just through the frozen in condition) in magnetic field generation, so that the field can be amplified by the CR pressure gradient. More importantly, this process amplifies the large scale field, required for acceleration of high energy particles. Furthermore, the amplification takes place well ahead of the gaseous subshock. The both requirements are crucial for improving high energy particle confinement and making the shock precursor shorter, in agreement with the observations. Large scales should be present in the ambient plasma as a seed for their amplification by the acoustic instability and could be driven (or seeded) by wave packet modulations. Apart from that, they result from the coalescence of shocks generated by the instability, and from the scattering of Alfven waves in $k$-space by these shocks to larger scales Malkov and Diamond (2006), Diamond and Malkov (2007), Malkov and Diamond (2009). Note that the Bell instability is essentially a short scale instability (the maximum growth rate is at scales smaller than the gyro-radii of accelerated particles). At larger scales the magnetic field growth rate is dominated by the modified resonant and the longwavelength nonresonant instabilities (Bvkov et al. 2011c). It should be noted that vorticity (and thus magnetic field) can be efficiently generated also at the subshock (see e.g. McKenzie and Westphal 1970, Bvkov 1982, 1988, Kevlahan 1997, Kulsrud et al. 1997, Giacalone and Jokipii 2007, Beresnyak et al. 2009, Fraschetti 2013). This would be too late for improving particle confinement and reducing the scale of the shock precursor. A more favorable for acceleration scenario is the above discussed field amplification in the CR shock precursor.

Now the question is which instability dominates the CR dynamics? Given the finite precursor crossing time, it is reasonable to choose the fastest growing mode and consider the development of a slower one under conditions created by the fast mode after its saturation. The Bell instability is likely to be efficient at the outskirt of the shock precursor where the CR current is dominated by the escaping CRs of the highest energies. The pressure gradient and the pitch angle anisotropy are strong enough to drive the acoustic and resonant instability in the shock precursor (see e.g. Pelletier et al. 2006). Recall that the anisotropy is typically inversely proportional to the local turbulence level which is usually decrease with the distance from the shock

Within the main part of the shock precursor, both the CR-pressure gradient and CR current are strong, so that the nonresonant CR-driven instabili- 
ties are likely to be the strongest candidates to govern the shock structure. In fact, these instabilities are coupled, not only by the common energy source but also dynamically. But first, it is important to identify conditions under which one of the instabilities dominates.

\section{Cosmic plasmas with cosmic rays: the governing equations}

In this section we discuss the governing equations for MHD-type flows of a cold background plasma interacting with cosmic rays. In most cases the cosmic ray particles are not subject to binary Coulomb or nuclear interactions with the background plasma particles. The interaction between the two components is due to both regular and fluctuating electromagnetic fields produced by the CRs. The momentum equation for the background plasma, including the Lorentz force associated with these fields is given by

$$
\widetilde{\rho}\left(\frac{\partial \widetilde{\mathbf{u}}}{\partial t}+(\widetilde{\mathbf{u}} \nabla) \widetilde{\mathbf{u}}\right)=-\nabla \widetilde{p}_{g}+\frac{1}{c} \widetilde{\mathbf{j}} \times \widetilde{\mathbf{B}}+e\left(\widetilde{n}_{p}-\widetilde{n}_{e}\right) \widetilde{\mathbf{E}}
$$

where $\widetilde{\mathbf{B}}$ is the magnetic field induction, $\widetilde{\mathbf{E}}$ - the electric field, $\widetilde{\mathbf{u}}$ - the bulk plasma velocity, $\widetilde{p}_{g}$ - the plasma pressure, $\widetilde{\mathbf{j}}$ - the electric current carried by the background plasma. We assume quasi-neutrality for the whole system consisting of background plasma protons of number density $\widetilde{n}_{p}$, electrons of number density $\widetilde{n}_{e}$, and cosmic rays of number density $\widetilde{n}_{c r}$. For simplicity we consider cosmic-ray protons only such that $\widetilde{n}_{p}+\widetilde{n}_{c r}=\widetilde{n}_{e}$, and typically $\widetilde{n}_{c r} \ll \widetilde{n}_{p}$.

The magnetic field is assumed to be frozen into the background plasma

$$
\widetilde{\mathbf{E}}=-\frac{1}{c}[\widetilde{\mathbf{u}} \times \widetilde{\mathbf{B}}]
$$

Both the background electric current $\widetilde{\mathbf{j}}$ and the electric current of accelerated particles $\widetilde{\mathbf{j}}^{c r}$ are the sources of magnetic fields in Maxwell's equations, where the Faraday displacement current was omitted for the slow MHD-type processes

$$
\nabla \times \widetilde{\mathbf{B}}=\frac{4 \pi}{c}\left(\widetilde{\mathbf{j}}+\widetilde{\mathbf{j}}^{c r}\right) .
$$

Then, for the quasi-neutral background plasmas, using Eq.(2), Eq.(3) and Eq.(4), one can write the induction equation and the equation of motion of the background plasma in the form used by Bell (2004), Bykov et al. (2011c), Schure and Bell 2011)

$$
\begin{gathered}
\frac{\partial \widetilde{\mathbf{B}}}{\partial t}=\nabla \times(\widetilde{\mathbf{u}} \times \widetilde{\mathbf{B}}) \\
\widetilde{\rho}\left(\frac{\partial \widetilde{\mathbf{u}}}{\partial t}+(\widetilde{\mathbf{u}} \nabla) \widetilde{\mathbf{u}}\right)=-\nabla \widetilde{p}_{g}+\frac{1}{4 \pi}(\nabla \times \widetilde{\mathbf{B}}) \times \widetilde{\mathbf{B}}-\frac{1}{c}\left(\widetilde{\mathbf{j}}^{c r}-e \widetilde{n}_{c r} \widetilde{\mathbf{u}}\right) \times \widetilde{\mathbf{B}}
\end{gathered}
$$


The microscopic CR-dynamics can be described by a kinetic equation for the single-particle distribution function $\tilde{f}$ that has the form

$$
\frac{\partial \tilde{f}}{\partial t}+\mathbf{v} \cdot \frac{\partial \tilde{f}}{\partial \mathbf{r}}+e \widetilde{\mathbf{E}} \cdot \frac{\partial \tilde{f}}{\partial \mathbf{p}}-\frac{e c}{\mathcal{E}} \widetilde{\mathbf{B}} \cdot \widehat{\mathcal{O}} \widetilde{f}=0
$$

where the $\mathrm{CR}$ particle energy is $\mathcal{E}, \widehat{\mathcal{O}}$ is the momentum rotation operator (see e.g. Toptygin 1983, Bykov et al. 2012). There are no Coulomb collisions in the kinetic equation Eq.(7), but the microscopic electromagnetic fields are fluctuating in a wide dynamical range due to collective plasma effects. The coarse grained distribution function of the CR particles $f=\langle\widetilde{f}>$ obeys the equation that can be obtained by averaging the microscopic equation Eq.(7) over an ensemble of appropriate short-scale fluctuations

$$
\frac{\partial f}{\partial t}+\mathbf{v} \cdot \frac{\partial f}{\partial \mathbf{r}}+e \mathbf{E} \cdot \frac{\partial f}{\partial \mathbf{p}}-\frac{e c}{\mathcal{E}} \mathbf{B} \cdot \widehat{\mathcal{O}} f=I\left[f, f^{\prime}\right] .
$$

Here $\widetilde{f}=f+f^{\prime}, \widetilde{\mathbf{B}}=\mathbf{B}+\mathbf{b}^{\prime}, \widetilde{\mathbf{E}}=\mathbf{E}+\mathbf{E}^{\prime}, \mathbf{B}=<\widetilde{\mathbf{B}}>, \mathbf{E}=<\widetilde{\mathbf{E}}>-$ are the averaged fields, and therefore $\left\langle\mathbf{b}^{\prime}\right\rangle=0,\left\langle\mathbf{E}^{\prime}\right\rangle=0$. The ensemble of fluctuations can be of external origin or produced by the same population of charged particles we only assumed at this point that the collision operator

$$
I\left[f, f^{\prime}\right]=-e\left\langle\mathbf{E}^{\prime} \cdot \frac{\partial f^{\prime}}{\partial \mathbf{p}}\right\rangle+\frac{e c}{\mathcal{E}}\left\langle\mathbf{b}^{\prime} \cdot \widehat{\mathcal{O}} f^{\prime}\right\rangle,
$$

is a functional of the averaged distribution function $f$ and can be expressed through the statistical momenta of the fluctuating field. The collision operator describes the momentum and energy exchange between CRs and the background plasma and therefore it must be accounted for in the averaged governing equations for both the CRs and background plasma.

The momentum exchange rate is the first moment of Eq.(9)

$$
\int \mathbf{p} I[f] d^{3} p=-e\left\langle n_{c r}^{\prime} \mathbf{E}^{\prime}\right\rangle+\frac{1}{c}\left\langle\mathbf{j}_{c r}^{\prime} \times \mathbf{b}^{\prime}\right\rangle,
$$

where $n_{c r}^{\prime}, \mathbf{j}_{c r}^{\prime}$ - are the fluctuating parts of the CR number density and the CRs electric current defined by

$$
\begin{gathered}
n_{c r}^{\prime}=e \int f^{\prime} d^{3} p, \\
\mathbf{j}_{c r}^{\prime}=e \int \mathbf{v}(p) f^{\prime} d^{3} p,
\end{gathered}
$$

where $\mathbf{v}(p)$ - is the CR particle velocity, and $\left\langle f^{\prime}\right\rangle=0$.

Then, by averaging the last term in Eq.(6), one can get

$$
\frac{1}{c}\left\langle\left(\widetilde{\mathbf{j}}_{c r}-e n_{\mathrm{cr}} \widetilde{\mathbf{u}}\right) \times \widetilde{\mathbf{B}}\right\rangle=\frac{1}{c}\left(\mathbf{j}^{c r}-e n_{c r} \mathbf{u}\right) \times \mathbf{B}-e\left\langle n_{c r}^{\prime} \mathbf{E}^{\prime}\right\rangle+\frac{1}{c}\left\langle\dot{\mathbf{j}}_{\mathrm{cr}}^{\prime} \times \mathbf{b}^{\prime}\right\rangle,
$$


where $n_{\mathrm{cr}}, \mathbf{j}_{\mathrm{cr}}$ - are the averaged CR number density and their electric current, $\widetilde{\mathbf{j}}_{c r}=\mathbf{j}_{\mathrm{cr}}+\mathbf{j}_{\mathrm{cr}}^{\prime}, \widetilde{n}_{c r}=n_{\mathrm{cr}}+n_{\mathrm{cr}}^{\prime}$. Note that Eq.(10) and the last two terms on the right hand side of Eq. (13) are coincident. Therefore, we conclude that the CR scattering due to the stochastic electromagnetic fields accounted for in the kinetic equation Eq. (8) by the collision operator must be simultaneously included into the equation of motion of the background plasma using Eq. (13).

The averaged induction equation Eq. (15) can be expressed as

$$
\frac{\partial \mathbf{B}}{\partial t}=\nabla \times(\mathbf{u} \times \mathbf{B})
$$

and the averaged equation of motion Eq.(6) for the background plasma

$\rho\left(\frac{\partial \mathbf{u}}{\partial t}+(\mathbf{u} \nabla) \mathbf{u}\right)=-\nabla p_{g}+\frac{1}{4 \pi}(\nabla \times \mathbf{B}) \times \mathbf{B}-\frac{1}{c}\left(\mathbf{j}^{c r}-e n_{\mathrm{cr}} \mathbf{u}\right) \times \mathbf{B}-\int \mathbf{p} I[f] d^{3} p$,

where $p_{g}$-is the averaged pressure of background plasma. Note that Eq. (14) and Eq. (15) is also valid for CRs consisting of electrons and positrons, with $n_{\mathrm{cr}}$ being the difference between the positron and the electron number densities, while $\mathbf{j}^{c r}$ - the total electric current of the particles.

In a few cases, namely, for weakly fluctuating magnetic fields or, for strong magnetic fluctuations but at scales smaller than the CR gyroradii, some closure procedures exist to reduce the collision operator $I\left[f, f^{\prime}\right]$ to $I[f]$ (see e.g. Toptygin 1983, Bykov et al. 2012). It is instructive, nevertheless, to derive the force density $\int \mathbf{p} I[f] d^{3} p$ for the most simple case of $I[f]$. The simplest form of the collision operator is the relaxation time approximation in the rest frame of the background plasma

$$
I[f]=-\nu\left(f-f_{\text {iso }}\right),
$$

where $f_{\text {iso }}$ - is the isotropic part of the momentum distribution $f$, and $\nu$ is the collision frequency due to CR particle-wave interactions (e.g. Bykov et al. 2011c). This approach usually implies that the scatterers have no mean (or drift) velocity relative to the rest frame of the background plasma. This is not always true, if the plasma instabilities that are producing the magnetic field fluctuations are highly anisotropic. However, it can be used to illustrate the importance of the momentum exchange between CRs and the background plasma.

Using the parameterisation $\nu=a \Omega$, where $\Omega=\frac{e c B_{0}}{\mathcal{E}}, \mathbf{B}_{0}$ is the mean magnetic field, and $a$ - is the CR collisionality parameter, from Eq.(16), one can obtain

$$
\int \mathbf{p} I[f] d^{3} p=-\frac{a B_{0}}{c} \mathbf{j}_{\mathrm{cr}} .
$$

This is the force density in Eq. (15). 


\section{Instabilities driven by anisotropic CR distributions: the kinetic approach}

Consider incompressible modes propagating along the mean homogeneous magnetic field $\mathbf{B}_{0}$ in the rest frame of the background plasma. The linear dispersion relation can be obtained by the standard perturbation analysis of Eq.(14), Eq.(15) and Eq.(8), assuming the small perturbations of magnetic field $\mathbf{b}$, plasma bulk velocity $\mathbf{u}$ and the CR distribution $f$ to be $\propto \exp (i k x-i \omega t)$. The unperturbed anisotropic CR distribution, that is the source of the instability free energy, can be represented as

$$
f_{0}^{c r}=\frac{n_{c r} N(p)}{4 \pi}\left[1+3 \beta \mu+\frac{\chi}{2}\left(3 \mu^{2}-1\right)\right],
$$

where $\mu=\cos \theta, \theta$ - is the CR particle pitch-angle, $n_{\mathrm{cr}^{-}}$CR number density. The multipole moments of the $\mathrm{CR}$ angular distribution are parameterized by $\beta$ (the dipole) and $\chi$ (the quadrupole). We assume below $\beta \leq 1$ and $\chi \leq 1$. The unperturbed state can be a steady state of a system with CRs where both the anisotropy and the spectral distribution $N(p)$ are determined by the energy source and sink as well as the magnetic field geometry through the kinetic equation Eq. (8) with some appropriate boundary conditions. The most interesting application of the formalism is related to diffusive shock acceleration model (see e.g. Blandford and Eichler 1987, Malkov and Drurv 2001, Bykov et al. 2012, Schure et al. 2012). In that case the normalized power-law CR spectrum is appropriate:

$$
N(p)=\frac{(\alpha-3) p_{0}^{(\alpha-3)}}{\left[1-\left(\frac{p_{0}}{p_{m}}\right)^{\alpha-3}\right] p^{\alpha}}, p_{0} \leq p \leq p_{m},
$$

where $\alpha$ - is the spectral index, $p_{0}$ and $p_{m}$ - are the minimal and maximal CR momenta, respectively. In the DSA applications it is convenient to express the dipole anisotropy parameters through the shock velocity $u_{\mathrm{s}}$ as $\beta=\frac{u_{\mathrm{s}}}{c}$.

Then dispersion equation has the form:

$$
\omega^{2}=v_{a}^{2}\left\{k^{2} \mp k\left[(1 \pm i a)\left(k_{0} A_{0}\left(x_{0}, x_{m}\right)+\frac{4 \pi e n_{c r} \chi}{B_{0}} A_{1}\left(x_{0}, x_{m}\right)\right)-k_{0}\right]\right\},
$$

where $v_{a}=\frac{B_{0}}{\sqrt{4 \pi \rho}}, k_{0}=\frac{4 \pi}{c} \frac{j_{0}^{c r}}{B_{0}}, j_{0}^{c r}=e n_{c r} u_{\mathrm{s}}, x=\frac{k c p}{e B_{0}}, x_{0}=\frac{k c p_{0}}{e B_{0}}$, $x_{m}=\frac{k c p_{m}}{e B_{0}}$,

$$
\begin{gathered}
A_{0,1}\left(x_{0}, x_{m}\right)=\int_{p_{0}}^{p_{m}} \sigma_{0,1}(p) N(p) p^{2} d p \\
\sigma_{0}(p)=\frac{3}{4} \int_{-1}^{1} \frac{\left(1-\mu^{2}\right)}{1 \mp x \mu \pm i a} d \mu, \\
\sigma_{1}(p)=\frac{3}{4} \int_{-1}^{1} \frac{\left(1-\mu^{2}\right) \mu}{1 \mp x \mu \pm i a} d \mu,
\end{gathered}
$$


where the \pm signs correspond to the two possible circular polarizations defined by $\mathbf{b}=b\left(\mathbf{e}_{y} \pm i \mathbf{e}_{z}\right)$, with the $x$-axis along the mean field $\mathbf{B}_{0}$. The functions $A_{0,1}\left(x_{0}, x_{m}\right)$ are expressed in elementary functions in Appendix A. In the collisionless limit $a \rightarrow 0$ the contribution of the pole to the imaginary part of Eq.(22) describes the well known resonant instability (e.g. Zweibel 1979, Amato 2011), while the real part (the principal part of the integral) is responsible for the instability discovered by Bell (2004) (see also Achterberg (1983)).

The kinetic approach we used here to derive the dispersion equation allows us to unify the instabilities due to both the dipole and quadrupoletype CR anisotropy. The finite mean free path of the CRs is characterized by the collisionality parameter $a$. The approach used above allows one to study the instabilities driven by the CR anisotropy for arbitrary relations between the mode wavelength, the CR mean free path and the CR gyroradii. It is instructive to demonstrate the transition between the collisionless case (i.e. $a=0$ ), where the $\mathrm{CR}$ mean free part is much larger than the mode wavelength, and the opposite case with the collisionality parameter $a \rightarrow 1$ (Bohm's diffusion limit). In the collisionless limit (i.e. $a=0$ ) the instabilities due to dipole type anisotropy $(\chi=0)$ were discussed by Bell (2004), Pelletier et al. (2006), and Amato and Blasi (2009). The firehose instability of a highly relativistic plasma without a dipole anisotropy was discussed by Noerdlinger and Yui (1968). Schure and Bell (2011) derived a dispersion equation for the mono-energetic particle distribution instead of the power-law distribution in Eq.(19) used here, and the dipole-type initial anisotropy (i.e. $\chi=0)$. The firehose instability of the anisotropic CR pressure with nonzero $\chi$ was studied by Bykov et al. (2011b).

\section{Growth rates of incompressible modes propagating along the mean magnetic field}

In Figure 1 we illustrate the growth rates derived from Eq. (20) for a particular choice of parameters of the CR distribution functions typical for the upstream distribution of CRs accelerated by the diffusive acceleration at a shock of velocity $\frac{u_{\mathrm{s}}}{c}=0.01$, with $\alpha=4$, and $\frac{p_{m}}{p_{0}}=100$. The DSA spectrum may span many decades, but we choose the two-decade range of the particle spectrum to model the instability far upstream of the shock where the longwavelength fluctuation amplification is the most efficient. The CR distribution function and the CR current normalizations are fixed here by the dimensionless parameter $k_{0} r_{g 0}=100$, where $r_{g 0}=\frac{c p_{0}}{e B_{0}}$. To estimate the normalization of the CR distribution we assumed that about $10 \%$ of the shock ram pressure is converted into the CR energy. For the CR spectrum of the index $\alpha=4$ the fraction of CRs above the momentum $p_{0}$ is $\propto p_{m} / p_{0}$, while $r_{g 0} \propto p_{0}$. Therefore the spatial dependence of the key governing parameter of the Bell instability $k_{0} r_{g 0}$ depends basically on the energy dependent CR anisotropy. The bulk of the CRs are confined in the accelerator and therefore 
would have anisotropy about $u_{\mathrm{s}} / c$ (apart from the particles at the very end of the CR spectrum escaping from the system).

\subsection{Nonresonant shortwavelength instability}

It is instructive to consider the short-scale CR-current driven modes produced by Bell's instability as an asymptotic case of the general Eq.(20), for different wavenumbers $k$ in the collisionless case $a=0$, following Bell (2004) and Bykov et al. (2011b). In Figure 1, we illustrate the growth rate dependence on the collisionality parameter.

In the wavenumber range $k_{0} r_{g 0}>k r_{g 0}>1$, corresponding to the instability discovered by Bell (2004), the growth of the right hand polarized mode (panel a in Figure 1) is much faster than the left hand mode (panel $b$ in Figure 11). This results in fast helicity production. In the collisionless limit the right hand mode has the growth rate

$$
\gamma_{b}=v_{a} \sqrt{k_{0} k-k^{2}} .
$$

Eq.(24) follows from Eq.(20), neglecting the response of the CR current on the magnetic fluctuations, i.e., $A_{0}\left(x_{0}, x_{m}\right) \rightarrow 0$ and $A_{1}\left(x_{0}, x_{m}\right) \rightarrow 0$. The weak CR-current response is the main cause of the Bell-type instability. Indeed, the CR current induces the compensatory reverse current in the background plasma and if the current is not responding to a magnetic field variation, then the magnetic fluctuation is growing due to the Ampere force. The CR current only weakly responds to the magnetic field fluctuations with wavenumbers $k_{0} r_{g 0}>k r_{g 0}>1$, and they grow. From Eq.(24) one may see that $\gamma_{b} \sim k^{\frac{1}{2}}$ for $k \ll k_{0}$.

\subsection{The resonant instability}

In the collisionless case for the wavenumber regimes $x_{m}>1$, but $x_{0}<1$, the resonant contribution dominates the pole in the integrand in Eq.(22). Therefore, the resonant mode growth can be seen in Figure 1 in the regime $0.01<k r_{g 0}<1$, where both circular polarization modes are growing with the very close rates $\propto k$ for $\alpha=4$ (compare panels (a) and (b) in Figure 1). Collisions do not change the mode growth drastically for $a<0.1$, but in the limit of strong collisions with $a=1$ the left hand mode grows slower than the right hand polarized mode. This may also result in helicity production.

4.3 A nonresonant longwavelength instability: the firehose mode

In the longwavelength regime where $x_{m}=\frac{k c p_{m}}{e B_{0}} \ll 1$, within the collisionless case, the dispersion relation in Eq.(20) can be approximated, following 


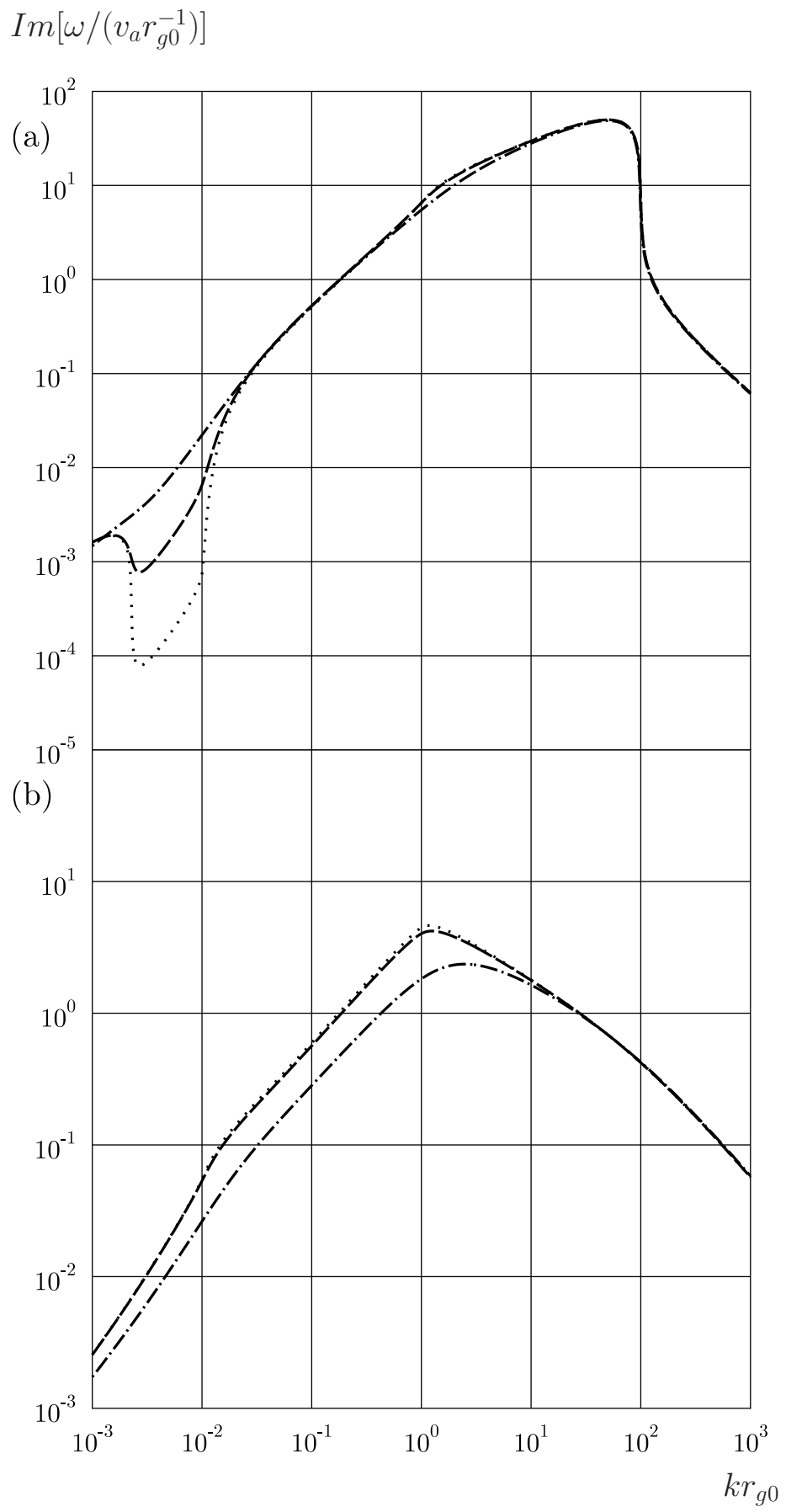

Fig. 1 The growth rates for the two circularly polarized modes. The right hand polarized mode (panel $a$ ) and the left hand mode (panel $b$ ) are derived from Eq.(20). We illustrate the growth rate dependence on the collisionality parameter $a$. Dotted line corresponds to $a=0.01$, dashed line $-a=0.1$, and dot-dashed line $-a=1$. The quadrupole anisotropy is $\chi=6\left(u_{\mathrm{s}} / c\right)^{2}$. Note that in the bottom panel the dashed and dotted lines are very close. 
Bykov et al. (2011b), as

$$
\omega^{2}=v_{a}^{2} k^{2}\left\{1 \mp \frac{r_{g 0}}{5}\left[k_{0} x_{m} \pm \frac{4 \pi e n_{c r} \chi}{B_{0}} \frac{\ln \frac{p_{m}}{p_{0}}}{\left(1-\frac{p_{0}}{p_{m}}\right)}\right]\right\} .
$$

As it follows from Eq.(25), in the regime dominated by the dipole CR anisotropy $(\chi \rightarrow 0)$ only the left-polarized mode is growing with the rate $\propto k^{\frac{3}{2}}$ (see Schure and Bell 2011). For a finite quadrupole-type CR anisotropy $\chi$ at small enough wavenumbers the modes of both circular polarizations are growing again with the very close rates $\propto k$ (see in Figure 1). The instability due to the quadrupole-type $\mathrm{CR}$ anisotropy corresponds to the well known firehose instability in a plasma with anisotropic pressure. Indeed, the CR pressure anisotropy derived from the CR distribution Eq.(18) is

$$
P_{\|}^{c r}-P_{\perp}^{c r}=\frac{3}{5} \chi P^{c r},
$$

where

$$
P^{c r}=\frac{1}{3} n_{c r} \int_{0}^{\infty} v(p) N(p) p^{3} d p .
$$

The dispersion relation for the modes produced by only the quadrupole-type anisotropy of CR distribution can be obtained from Eq.(25) if one neglects the dipole-type contribution $x_{m} \rightarrow 0$. Then, it is reduced to the standard hydrodynamic dispersion relation of the firehose instability

$$
\omega= \pm \sqrt{v_{a}^{2}-\frac{P_{\|}-P_{\perp}}{\rho}} k,
$$

where $P_{\|}-P_{\perp}$ - is the pressure anisotropy along the mean magnetic field direction (see, e.g., Blandford and Eichler 1987, Treumann and Baumjohann 1997). The dispersion relation Eq.(28) is justified for the modes with the wavenumbers above the $\mathrm{CR}$ ion gyroradii. The dependence of the growth rates of the firehouse instability on the collisionality parameter can be seen in Figure 1 It should be noted that the growth rates of the firehose modes of both polarizations in the regime $k r_{g 0}<1$ are declining functions of the collisionality parameter. Their growth rates would be equal in the case of lack of the CR current. Contrary, the growth rates of the current driven modes are different for the two polarizations. The growth rate of the right hand polarized CR-current driven mode is sensitive to the collisionality parameter (see Schure and Bell 2011).

4.4 A nonresonant long-wavelength instability: the cosmic-ray current driven dynamo

Bell's instability results in the fast growth of short-scale modes with wavelengths shorter than the gyroradius of the cosmic-ray particles and in the presence of CR-current it may produce strong short-scale turbulence (e.g. 


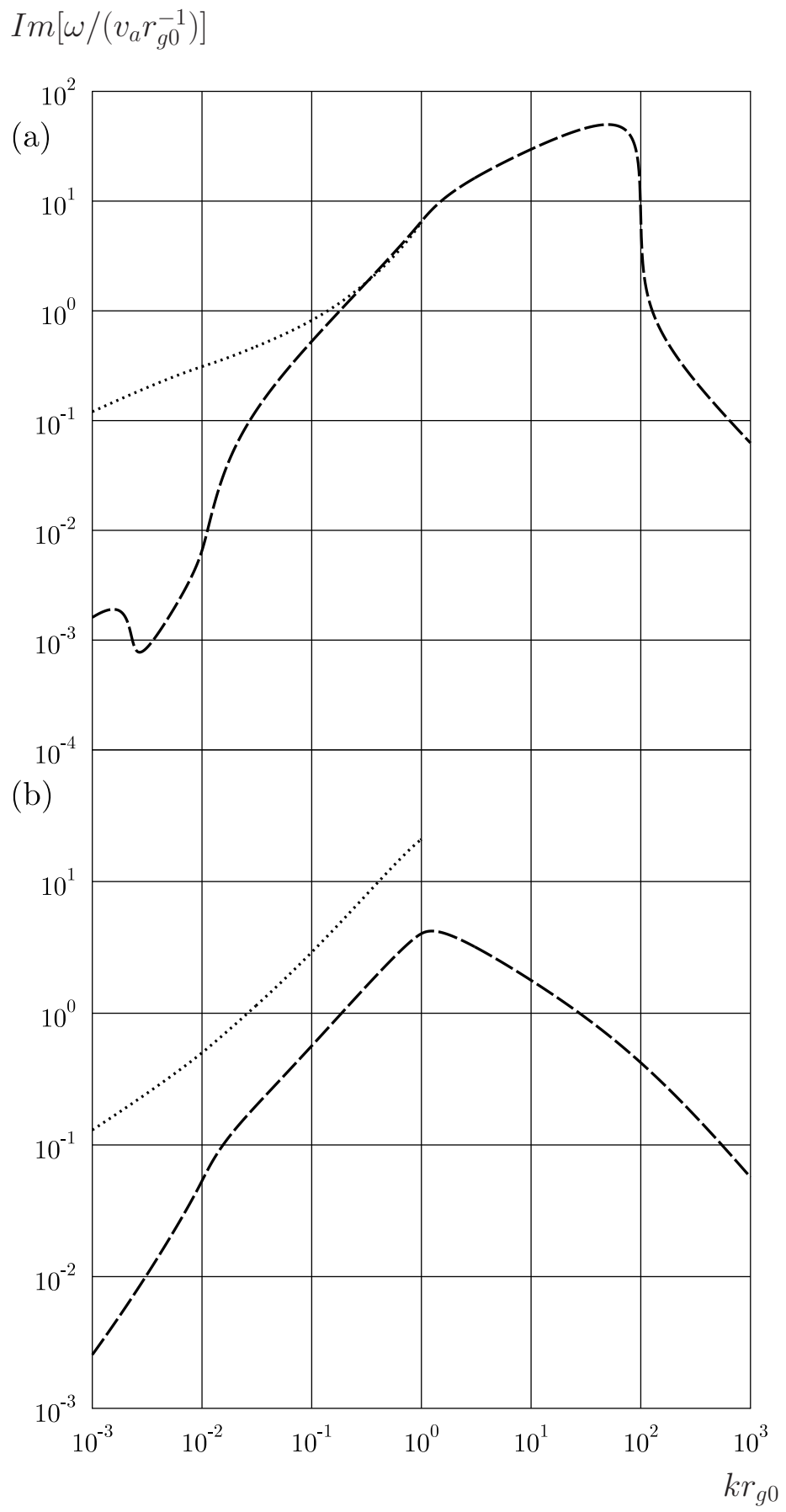

Fig. 2 The growth rates of the longwavelength modes of two circular polarizations. The right hand polarized mode (panel $a$ ) and the left hand mode (panel $b$ ) are propagating along the mean magnetic field as function of the wavenumber. The dotted line curves are derived from the dispersion equation Eq.(31) for the collisionality parameter $a=0.1$, the dimensionless r.m.s. amplitude of Bell's turbulence $N_{B}=1$, and the mixing parameter $\xi=3$. The dashed curves given for comparison are the growth rates derived from Eq. (20) which are shown in Figure 1 
Bell and Lucek 2001, Bell 2004, Zirakashvili and Ptuskin 2008, Zirakashvili et al. 2008, Reville et al. 2008, Vladimirov et al. 2009, Rogachevskii et al. 2012). Moreover, the shortscale turbulence is helical, and at the wavenumbers below $2 k_{0}$ its kinetic energy density dominates over the magnetic energy density making a favorable condition for a pure $\alpha$-dynamo effect (see Bykov et al. 2011c). The strong short-scale turbulence influences the background plasma dynamics on scales larger than the CR gyroradii. Bykov et al. (2011c) derived the mean field dynamic equations averaged over the ensemble of short-scale motions for plasma systems with CR-current. The averaged equation of motion can be presented as

$$
\begin{aligned}
& \frac{\partial \mathbf{V}}{\partial t}+(\mathbf{V} \nabla) \mathbf{V}=-\frac{1}{\rho} \nabla P_{g}-\langle(\mathbf{u} \nabla) \mathbf{u}\rangle+\frac{1}{4 \pi \rho}\langle(\nabla \times \mathbf{b}) \times \mathbf{b}\rangle+ \\
& +\frac{1}{4 \pi \rho}((\nabla \times \mathbf{B}) \times \mathbf{B})--\frac{1}{c \rho}\left(\left(\mathbf{j}^{\mathrm{cr}}-e n_{\mathrm{cr}} \mathbf{V}\right) \times \mathbf{B}\right)-\int \mathbf{p} I[f] d^{3} p,
\end{aligned}
$$

where $\mathbf{V}$ is the mean velocity of the plasma. The magnetic induction equation for the mean magnetic field $\mathbf{B}$ reads

$$
\frac{\partial \mathbf{B}}{\partial t}=c \nabla \times \overline{\mathcal{E}}+\nabla \times(\mathbf{V} \times \mathbf{B})+\nu_{m} \triangle \mathbf{B} .
$$

Here $\overline{\mathcal{E}}=\langle\mathbf{u} \times \mathbf{b}\rangle$ is the average turbulent electromotive force and $\nu_{m}$ is the magnetic diffusivity. The averaged equations Eq.(29) and Eq.(30) are designed to be applied to the dynamics of modes with scales larger than $r_{g 0}$, i.e., $\mathrm{CR}$ particles are magnetized on these scales.

The ponderomotive forces $\langle(\mathbf{u} \nabla) \mathbf{u}\rangle$ and $\frac{1}{4 \pi \rho}\langle(\nabla \times \mathbf{b}) \times \mathbf{b}\rangle$ in Eq.(29) describe the momentum exchange of the background plasma with the Bell mode turbulence. The averaged turbulent electromotive force $\overline{\mathcal{E}}$ results in the magnetic induction evolution. It is important that in the case under consideration the ponderomotive forces in Eq. (29) depend on the CR current through the Bell mode turbulence moments. To express the electromotive and ponderomotive forces through the CR current Bvkov et al. (2011c) followed the mean field closure procedure similar to the approach proposed by Blackman and Field (2002) in the dynamo theory (see for a review Brandenburg 2009a). The closure procedure is introduced by the parameter $\tau_{\text {cor }}$. The correlation time $\tau_{\text {cor }}$ which is the relaxation time of triple correlations and is approximately equal to the turnover time of the Bell turbulence. The dependence of the electromotive force and the ponderomotive force on the $\mathrm{CR}$ current is determined by the kinetic coefficients $\alpha_{t}$ and $\kappa_{t}$, correspondingly. The kinetic coefficients are determined by the r.m.s. amplitude of Bell's turbulence $\left\langle b_{B}^{2}\right\rangle$ and $\tau_{\text {cor }}$. The short scale turbulence produced by the Bell mode instability is helical and therefore there is also a contribution to the electromotive force $\propto \alpha_{t} \bar{B}$ resulting in the $\alpha$-dynamo effect. Then, the dispersion equation for the modes of wavelengths longer than $r_{g 0}$ in a plasma with anisotropic relativistic CRs can be derived from Eq.(29) and Eq.(30) by the standard linear perturbation analysis:

$$
\omega^{2}-k^{2} v_{a}^{2} \mp \omega i k \frac{\alpha_{t}}{4 \pi \rho}\left[\frac{1}{2}\left(k_{0} A_{0}\left(x_{0}, x_{m}\right)+\frac{4 \pi e n_{c r} \chi}{B_{0}} A_{1}\left(x_{0}, x_{m}\right)\right)+\frac{3}{2} k_{0}\right]
$$




$$
\begin{aligned}
& \pm k v_{a}^{2}\left(1+\frac{\kappa_{t}}{B_{0}}\right)\left[\left(k_{0} A_{0}\left(x_{0}, x_{m}\right)+\frac{4 \pi e n_{c r} \chi}{B_{0}} A_{1}\left(x_{0}, x_{m}\right)\right)-k_{0}\right] \\
& +i a k v_{a}^{2}\left(k_{0} A_{0}\left(x_{0}, x_{m}\right)+\frac{4 \pi e n_{c r} \chi}{B_{0}} A_{1}\left(x_{0}, x_{m}\right)\right)=0 .
\end{aligned}
$$

The dispersion relation Eq. (31) was derived for the systems where the unperturbed CR-current is directed along the unperturbed magnetic field, and the short scale turbulence consists of Bell's modes. It is convenient to introduce two dimensionless parameters $N_{B}=\frac{\sqrt{\left\langle b_{B}^{2}\right\rangle}}{B_{0}}-$ Bell's turbulence r.m.s. amplitude, and the dimensionless mixing length $\xi$, instead of the correlation time $\tau_{\text {cor. }}$. The mixing length is defined here as $2 \pi \xi / k_{0}=\tau_{\text {cor }} \sqrt{\left\langle v^{2}\right\rangle} \approx$ $\tau_{\text {cor }} \sqrt{\xi\left\langle b_{B}^{2}\right\rangle /(4 \pi \rho)}$. Then $\alpha_{t} \approx\left\langle b_{B}^{2}\right\rangle \tau_{\text {cor }} \approx 8 \pi^{2} \sqrt{\xi} N_{B} v_{a} k_{0}^{-1} \rho$ and $\kappa_{t}=\pi N_{B} B_{0}$.

In Figure 2 we illustrate the long wavelength mode growth derived from Eq. (31) for $\xi=3$. The corresponding mixing length is close to the scale of the maximal growth rate of the short scale Bell's instability. The $\alpha$-dynamo effect dominates the growth rate of a polarized mode shown in Figure 2 (panel b) in the intermediate wavenumber regime $a<k r_{g 0}<1$. One should have in mind that in the case of Bohm's CR diffusion $a \sim 1$ and therefore the intermediate wavenumber regime is rather limited. It should be noted that the helicity of the unstable, long-wavelength mode studied above is opposite to that of the short-scale Bell mode. This provides, at least in principle, the possibility of balancing the global helicity of the system by combining short and long-wavelength modes. Care must be taken however, since numerical models indicate a high saturation amplitude of the Bell mode making a nonlinear analysis necessary to address the helicity balance issue. We will discuss some nonlinear simulations below in $\$ 5$.

4.5 The cosmic-ray current driven instability in the hydrodynamic regime

The nonresonant modes in a hydrodynamic regime, where the wavelength is longer than the mean free path, i.e., $k r_{g 0}<a$, are unstable, as it follows from Eq.(31) (see Bykov et al. 2011c, for details). Both circular polarizations in panels $a$ and $b$ in Figure 2 grow with the same rate given by

$$
\gamma \approx \sqrt{\frac{\pi N_{B}}{2}} \sqrt{k k_{0} a} v_{a} .
$$

The transition from the intermediate wavenumber regime $a<k r_{g 0}<1$, dominated by the dynamo effect discussed in 4.4 where the mode growth rate can be approximated by

$$
\gamma \approx 4 \pi \sqrt{\xi} N_{B} v_{a} k
$$

to the hydrodynamical regime with $k r_{g 0}<a$ where the growth rate is $\propto k^{1 / 2}$ according to Eq.(32), is clearly seen in Figure 2 (panel $b$ ). Note that for the mode polarization shown with the dotted line in the panel $a$ of Figure 2, no dynamo-type instability occurs, but the hydrodynamical regime instability 
is present. This mode grow fast in the short wavelength regime $k r_{g 0}>1$ due to Bell's instability.

The effect of the short-scale turbulence on the hydrodynamic regime instability enters Eq.(31) through the turbulent coefficient $\kappa_{t} / B_{0}$. The turbulent ponderomotive force is large enough in both the intermediate and hydrodynamical regimes, and the $\mathrm{CR}$ current response in the long-wavelength regime can no longer be neglected. The current cannot be treated as a fixed external parameter, as is normally done for the short-scale Bell instability, and therefore the MHD models of the Bell turbulence that assume a constant CR-current (see e.g. Bell and Lucek 2001, Zirakashvili and Ptuskin 2008, Zirakashvili et al. 2008, Reville et al. 2008, Vladimirov et al. 2009, Rogachevskii et al. 2012) cannot be directly applied to the nonlinear models of the longwavelength instabilities discussed above. Particle-in-cell simulations with very limited dynamical range performed by Riquelme and Spitkovskv (2009, 2010) indicate the importance of the CR backreaction effect on the CR-driven instabilities. Therefore the nonlinear dynamics of the long-wave CR-driven turbulence in a wide dynamical range remains to be investigated. In the next section we illustrate the nonlinear evolution of the short scale turbulence driven by a fixed CR current, using high resolution MHD simulations.

\section{Numerical solutions of the Bell-dynamo instability}

Significant insights have been possible through high-resolution direct numerical simulations (DNS) and large eddy simulations (LES) of the Bell instability and its subsequent saturation. In this section we describe some of the main results and, in particular, the connection with the dynamo instability. The simulations have been carried out in a Cartesian domain of size $L^{3}$, so the smallest wavenumber in that domain is $k_{1}=2 \pi / L$. The system is characterized by the non-dimensional parameter

$$
\mathcal{J}=\frac{4 \pi}{c} \frac{j^{\mathrm{cr}}}{k_{1} B_{0}} .
$$

In the ideal case $\left(\nu_{M}=0\right)$, the Bell instability is excited when $\mathcal{J}>1$ and the normalized wavenumber of the fastest growing mode is $k / k_{1}=\mathcal{J} / 2$. The normalized growth rate of this fastest growing mode is $\gamma_{b} / v_{A 0} k_{1}=$ $\mathcal{J} / 2$. In Figure 3 we reproduce the results of numerical simulations of Bell (2004) for $\mathcal{J}=2$ using $128^{3}$ mesh points and Zirakashvili et al. (2008) for $\mathcal{J}=16$ using $256^{3}$ mesh points. These simulations confirmed the analytically expected linear growth rates. Interestingly, the saturation of the instability was never perfect. Instead, the magnetic field still continued to grow at a slow rate. Rogachevskii et al. (2012) have argued that this slow growth after the end of the exponential growth phase of the instability is the result of a mean-field $\alpha$ effect. The purpose of this section is to elaborate on this possibility.

We begin by discussing first the recent DNS of Rogachevskii et al. (2012) for $\mathcal{J}=80$ and $\mathcal{J}=800$ at a resolution of $512^{3}$ mesh points and discuss also new results for $\mathcal{J}=800$ at a resolution of $1024^{3}$ mesh points. In all 
cases, explicit viscosity $\nu$ and magnetic diffusivity $\nu_{\mathrm{M}}$ are used, so the fastest growing modes in those cases have somewhat smaller wavenumbers than in the ideal case. This is quantified by the Lundquist number $\mathrm{Lu}=v_{a} / \nu_{\mathrm{M}} k_{1}$ and the ideal case corresponds then to $\mathrm{Lu} \rightarrow \infty$. For example, Rogachevskii et al. (2012) used $\mathrm{Lu}=80$, in which case the fastest growing mode has $k_{z} / k_{1} \approx 21$ for $\mathcal{J}=80$ while for $\mathcal{J}=800$ it has $k_{z} / k_{1} \approx 63$. The DNS show that most of the power is at somewhat larger wavenumbers; see Figure 4, where we show magnetic energy spectra for both cases.

In Figure 5 we show the temporal evolution of spectral magnetic energy $E_{M}$ and the spectral kinetic energy $E_{K}$ at selected wavenumbers. These curves show an exponential growth at early times, followed by a slower growth at later times. At the wavenumbers of the Bell mode, the growth rate from linear theory is reproduced. At smaller wavenumbers, the growth is at first slower, and then it is even faster than the growth rate of the Bell mode. This is
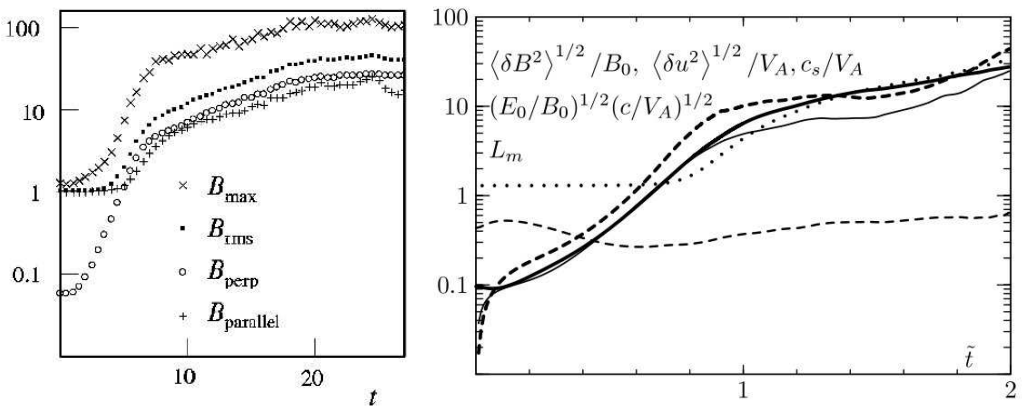

Fig. 3 Numerical solutions of the Bell instability for $\mathcal{J}=2$ using $128^{3}$ mesh points (Bell 2004, left hand side) and $\mathcal{J}=16$ using $256^{3}$ mesh points (Zirakashvili et al. 2008, right hand side). Note the continued growth of the magnetic field at the end of the linear growth phase at $t \approx 10$ on the left and $t \approx 1$ in the right. Courtesy of Tony Bell (left panel) and Vladimir Zirakashvili (right panel).
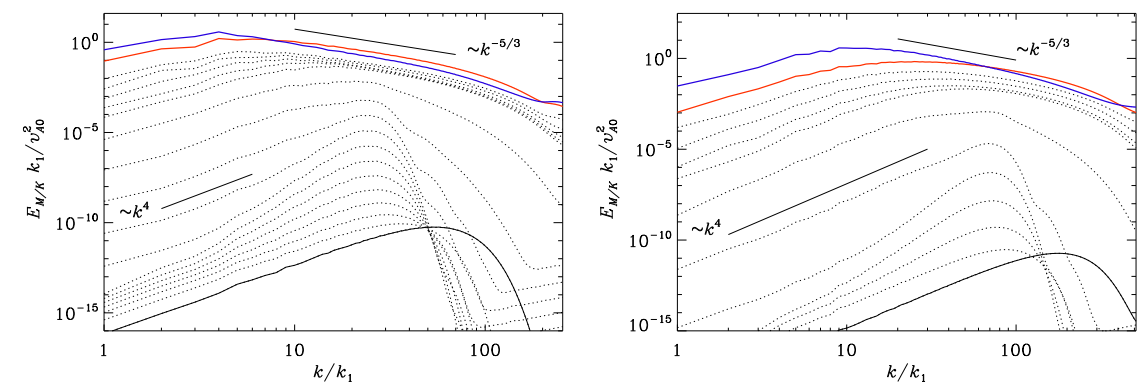

Fig. 4 Time evolution of $E_{M}(k, t)$ for $\mathcal{J}=80$ (left) and $\mathcal{J}=800$ (right) at resolutions $512^{3}$ and $1024^{3}$, respectively. The solid lines refer to the initial spectra proportional to $k^{4}$ for small values of $k$ and the red and blue lines represent the last instant of $E_{M}$ and $E_{K}$, respectively. The straight lines show the $k^{4}$ and $k^{-5 / 3}$ power laws. 

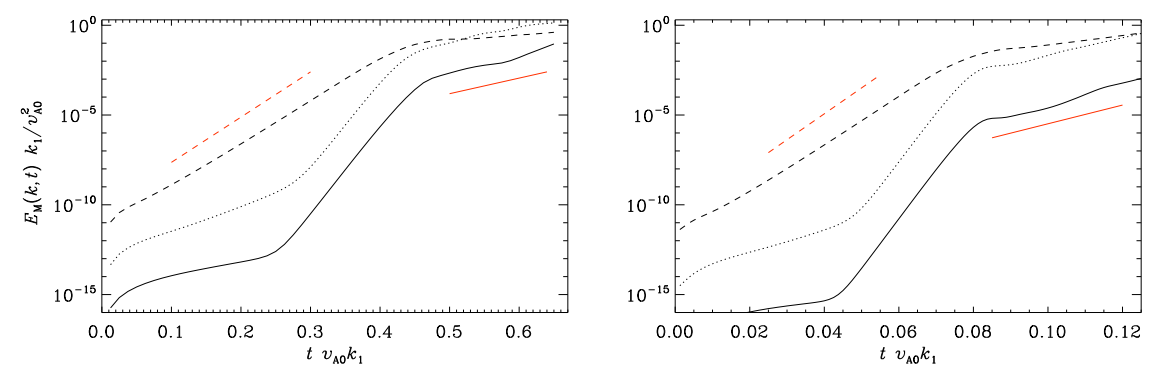

Fig. 5 Time evolution of $E_{M} k_{1} / v_{A 0}^{2}$ for $\mathcal{J}=80$ (left) at wavenumbers $k / k_{1}=1$ (solid line), 5 (dotted), and 21 (dashed) and $\mathcal{J}=800$ (right) at wavenumbers $k / k_{1}=1$ (solid line), 10 (dotted), and 63 (dashed). The short straight lines show the growth of the energies for the Bell (dashed) and dynamo (solid) instabilities.

a consequence of mode coupling (Rogachevskii et al. 2012). Comparing with Figure 4 we see that after some time a $k^{4}$ energy spectrum is established. Such an energy spectrum is also known as Batchelor spectrum and can be derived under the constraints of solenoidality and causality (Durrer and Caprini 2003). When the $k^{4}$ spectrum is established, the growth of spectral energy at small wavenumbers is no longer described by linear theory, but follows the growth of the Bell mode.

In Figure 6 we show visualizations of $B_{x} / B_{0}$ on the periphery of the computational domain for $\mathcal{J}=80$ using $512^{3}$ mesh points and $\mathcal{J}=800$ using $1024^{3}$ mesh points at two different times. One clearly sees that at early times, the magnetic field shows a layered structure with a high wavenumber in the $z$ direction. At later times, the magnetic field breaks up and becomes turbulent. In both cases, larger scale structures develop, as one also sees from the energy spectra in Figure 4

It should be pointed out that, owing to the persistent growth of magnetic and kinetic energy, the Reynolds numbers grow eventually beyond the limit of what can be resolved at a given resolution. Unlike some of the earlier LES, where numerical effective viscosity and diffusivity keep the small scales resolved, in the DNS of Rogachevskii et al. (2012) this is not the case and the numerical code (in this case the PencIL Code1) eventually 'crashes'. The point when this happens can be delayed by using higher resolution. This is why we show here the results for $\mathcal{J}=800$ at a resolution of $1024^{3}$ mesh points, where the simulation can be carried out for about 0.126 Alfvén times, compared to only 0.09 Alfvén times at a resolution of $512^{3}$ mesh points used in Rogachevskii et al. (2012). Remeshing the $1024^{3}$ run to $2048^{3}$ mesh points, we were able to continue until 0.142 Alfvén times, after which we were unable to continue the run due to a disk problem.

The Bell instability is driven by the simultaneous presence of an external magnetic field $\mathbf{B}_{0}$ and an external current $\mathbf{j}_{\mathrm{cr}}$, giving therefore rise to a pseudo-scalar $\mathbf{j}_{\text {cr }} \cdot \mathbf{B}_{0}$; here, $\mathbf{B}_{0}$ is an axial vector while $\mathbf{j}_{\text {cr }}$ is a polar vector. In stellar magnetism, the presence of a pseudo-scalar is caused by rotation

${ }^{1}$ http://www.pencil-code.googlecode.com 

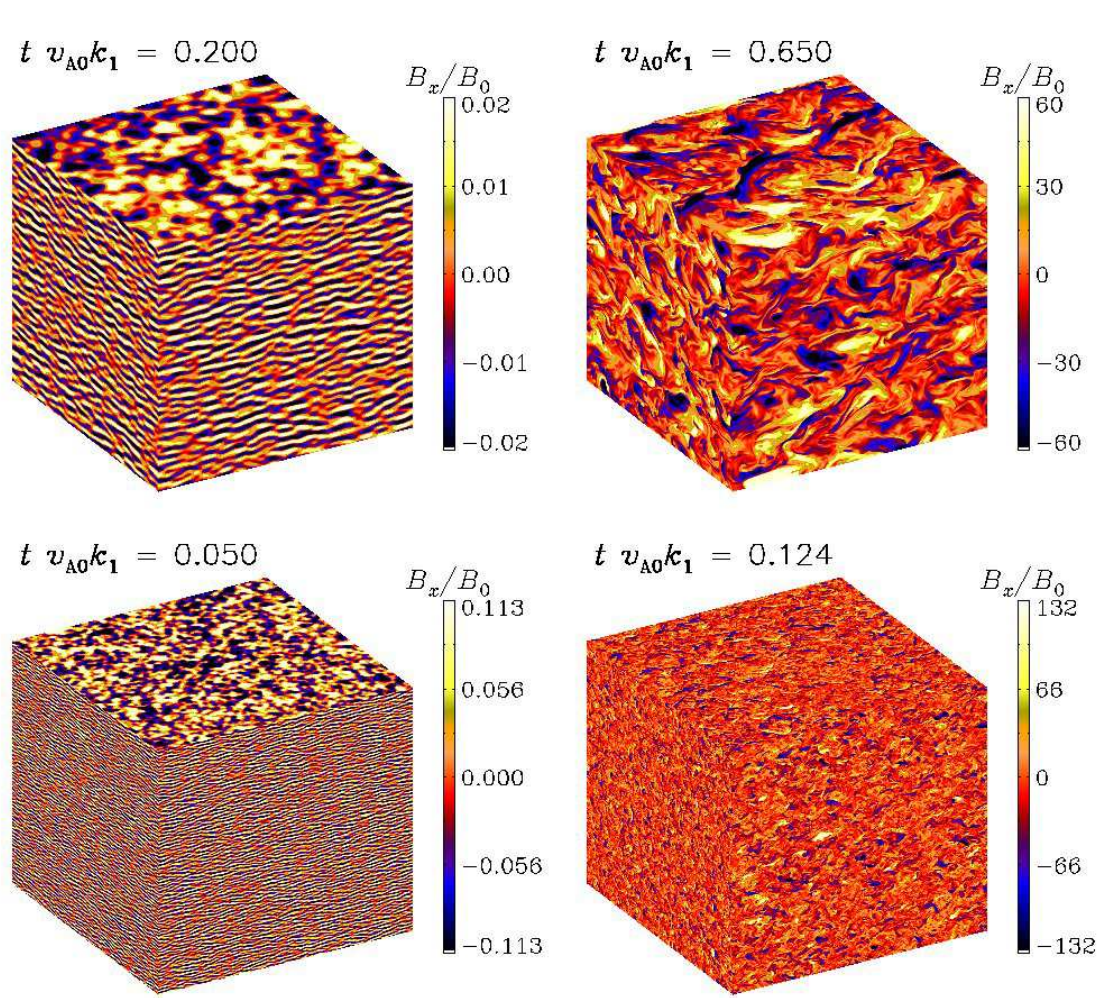

Fig. 6 Visualization of $B_{x} / B_{0}$ on the periphery of the computational domain $\mathcal{J}=80$ using $512^{3}$ mesh points (upper row) and $\mathcal{J}=800$ using $1024^{3}$ mesh points (lower row) with Lundquist number $\mathrm{Lu}=80$ in both cases.

$\boldsymbol{\Omega}$ (an axial vector) and gravity $\mathrm{g}$ (a polar vector). This property is generally held responsible for the production of magnetic fields by what is known as the $\alpha$ effect. As explained in Sect. 4.4, the $\alpha$ effect denotes the presence of a tensorial connection between a mean electromotive force $\overline{\mathcal{E}}=\overline{\mathbf{u} \times \mathbf{b}}$ and a mean magnetic field via

$$
\overline{\mathcal{E}}_{i}=\alpha_{i j} \bar{B}_{j}+\eta_{i j k} \bar{B}_{j, k}+\ldots,
$$

where higher order derivatives (indicated by commas) of the mean magnetic field are also present. If the tensors $\alpha_{i j}$ and $\eta_{i j k}$ were isotropic and the evolution characterized by just two quantities, $\alpha=\delta_{i j} \alpha_{i j} / 3$ and $\eta_{t}=\epsilon_{i j k} \eta_{i j k} / 6$, the growth of the mean magnetic field would occur at the rate

$$
\gamma_{\text {dynamo }}=\alpha k-\eta_{T} k^{2}
$$

where $\eta_{T}=\eta_{t}+\nu_{M}$ is the total (turbulent plus microphysical) magnetic diffusivity and the fastest growth occurs at wavenumber $k=\alpha / 2 \eta_{T}$ with the growth rate $\gamma_{\max }=\alpha^{2} / 4 \eta_{T}$. 
In stellar dynamos, where the magnetic Reynolds number is very large, the actual growth is dominated by small-scale dynamo action, so Eq. (36) is in practice not obeyed, unless the small-scale dynamo is not excited, for example at low magnetic Prandtl numbers (Brandenburg 2009b). However, in the present case the magnetic energy spectra show that at late times, magnetic power moves gradually to larger scales. This is why we now ask whether this can be explained by the $\alpha$ effect.

Rogachevskii et al. (2012) have shown that in the case of $\mathbf{j}_{\mathrm{cr}}$ and $\mathbf{B}_{0}$ pointing in the $z$ direction, the large-scale mean magnetic field is a function of $x$ and $y$ and can be written in terms of two scalar functions $\bar{A}_{\|}(x, y, t)$ and $\bar{B}_{\|}(x, y, t)$ with

$$
\overline{\mathbf{B}}(x, y, t)=\nabla \times\left(\hat{\mathbf{z}} \bar{A}_{\|}\right)+\hat{\mathbf{z}} \bar{B}_{\|},
$$

where $\hat{\mathbf{z}}=(0,0,1)$ is the unit vector in the $z$ direction. These functions obey the mean field equations

$$
\begin{gathered}
\partial \bar{A}_{\|} / \partial t=\alpha_{A} \bar{B}_{\|}+\eta_{A} \nabla^{2} \bar{A}_{\|}, \\
\partial \bar{B}_{\|} / \partial t=\alpha_{B} \bar{J}_{\|}+\eta_{B} \nabla^{2} \bar{B}_{\|},
\end{gathered}
$$

where $\bar{J}_{\|}=-\nabla^{2} \bar{A}_{\|}$is the $x y$ dependent part of the mean current density in the $z$ direction. We consider a homogeneous system, so the coefficients $\alpha_{A}$, $\alpha_{B}, \eta_{A}$, and $\eta_{B}$ are constant and we can seek solutions of a form proportional to $\exp (\lambda t+\mathrm{ik} \cdot \mathbf{x})$. In this case, the dynamo growth rate is still described by Eq.(36), provided we substitute

$$
\alpha \rightarrow \alpha^{\mathrm{eff}}=\left(\alpha_{A} \alpha_{B}+\epsilon_{\eta}^{2} k^{2}\right)^{1 / 2} \quad \text { and } \quad \eta_{t} \rightarrow \eta_{t}^{\mathrm{eff}}=\left(\eta_{A}+\eta_{B}\right) / 2,
$$

where $\epsilon_{\eta}=\left(\eta_{A}-\eta_{B}\right) / 2$ quantifies the anisotropy of the turbulent diffusivity.

To determine these coefficients from the DNS, we use the so-called testfield method of Schrinner et al. (2005), which was originally used in spherical coordinates. The implementation in Cartesian coordinates is described in Brandenburg (2005) and especially in Brandenburg et al. (2012), where the mean magnetic field was allowed to depend on all three spatial coordinates, and not just on one, as was assumed in Brandenburg (2005). Under the assumption that the turbulence is governed by only one preferred direction, which is here the case, the number of coefficients reduces to 9 , and homogeneity reduces this number further to 5 , so in the present case we have

$$
\overline{\mathcal{E}}=\alpha_{\perp} \overline{\mathbf{B}}_{\perp}+\alpha_{\|} \overline{\mathbf{B}}_{\|}-\beta_{\perp} \overline{\mathbf{J}}_{\perp}+\beta_{\|} \overline{\mathbf{J}}_{\|}-\mu \hat{\mathbf{z}} \times \overline{\mathbf{K}}_{\perp},
$$

where $\overline{\mathbf{J}}=\nabla \times \overline{\mathbf{B}}$ characterizes the antisymmetric part of the magnetic derivative tensor and $\bar{K}_{i}=\left(\bar{B}_{i, j}+\bar{B}_{i, i}\right) \hat{z}_{i} / 2$ the symmetric part. We have followed here the notation of Brandenburg et al. (2012), except that there the two $\alpha$ coefficients were defined with the opposite sign. Comparing with the coefficients used in Eqs. (38) and (39), we find that $\alpha_{A}=\alpha_{\|}, \alpha_{B}=\alpha_{\perp}, \eta_{A}=\beta_{\|}$, and $\eta_{B}=\beta_{\perp}-\mu / 2$. In Figure 7 we show the time dependence of the various parameter combinations. In the early kinematic phase $\left(t v_{\mathrm{A} 0} k_{1}<0.08\right)$, the root mean square velocity, $u_{\mathrm{rms}}$, as well as $\alpha_{\|}$and $\alpha_{\perp}$ grow exponentially. At later times, $\alpha_{\|}$continues to grow, while $\alpha_{\perp}$ remains small and approximately 

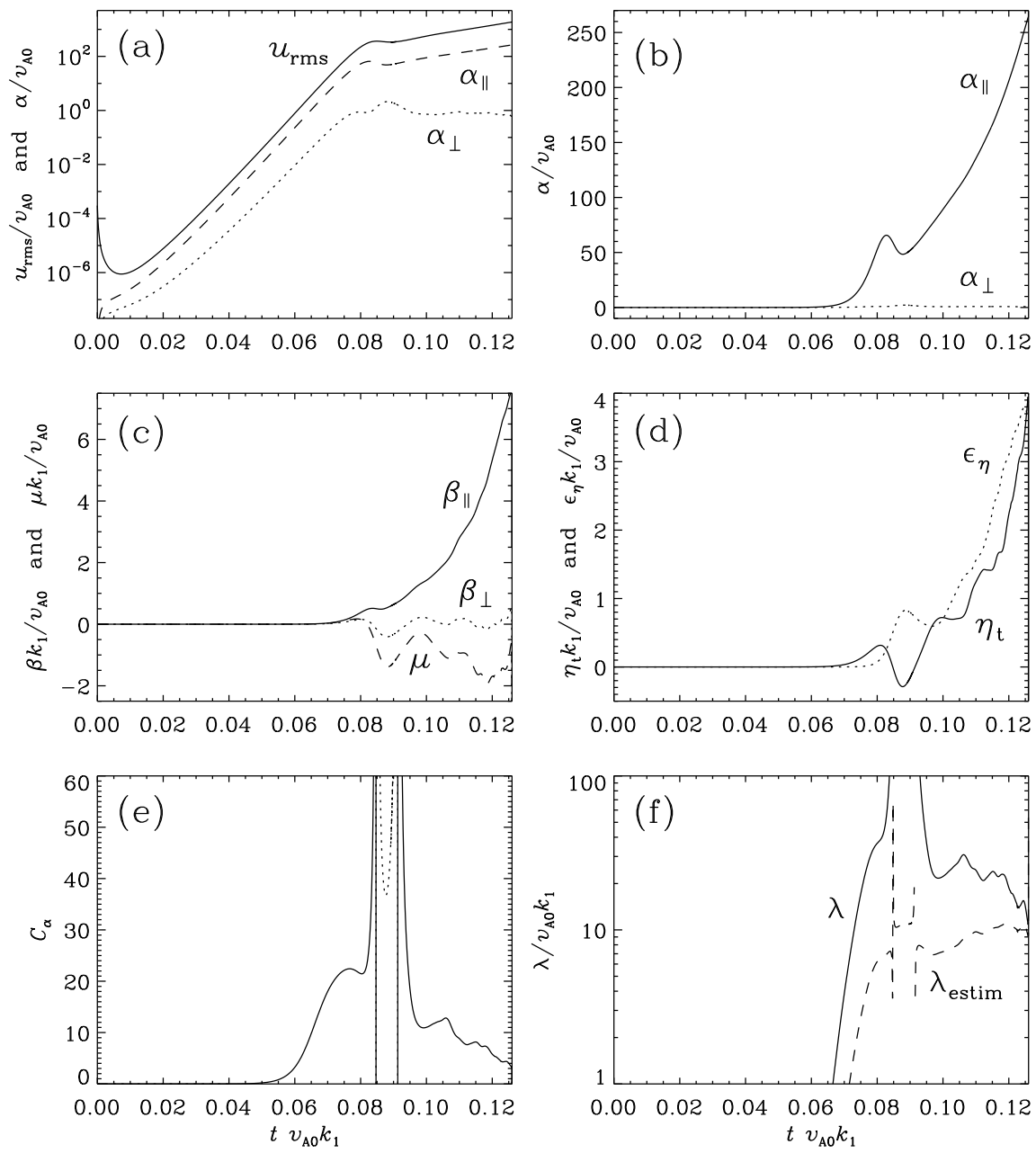

Fig. 7 Time evolution of the model parameters for $\mathcal{J}=800$ and $\mathrm{Lu}=80$ using $1024^{3}$ mesh points. (a) Exponential growth and subsequent near-saturation of $u_{\mathrm{rms}}$, $\alpha_{\|}$, and $\alpha_{\perp}$ (all normalized by $v_{\mathrm{A} 0}$ ) in linear-logarithmic representation. (b) Evolution of $\alpha_{\|}$and $\alpha_{\perp}$ (normalized by $v_{\mathrm{A} 0}$ ) in double linear representation, showing that $\alpha_{\perp}$ is much smaller than $\alpha_{\|}$. (c) Evolution of $\beta_{\|}, \beta_{\perp}$, and $\mu$ (normalized by $\left.v_{\mathrm{Ao}} / k_{1}\right)$. (d) Evolution of $\eta_{\mathrm{t}}$ and $\epsilon_{\eta}$ (normalized by $v_{\mathrm{A} 0} / k_{1}$ ). (e) Evolution of $C_{\alpha}$ (negative values are shown as dotted lines), and (f) growth of the fasted growing mode.

constant. The other turbulent transport coefficients also grow exponentially in the kinematic phase, and at later times $\beta_{\|}, \eta_{t}$, and $\epsilon_{\eta}$ continue to grow, while $\beta_{\perp}$ and $\mu$ remain small and can even become negative. The resulting effective dynamo number, which is proportional to the product $\alpha_{\|} \alpha_{\perp}$, reaches values well above the critical value of unity. The estimated and actual growth rates agree roughly and have a value of around 10 in units of $\eta_{t} k_{1}$. 


\section{Instabilities driven by the nearly isotropic CR distributions}

In many astrophysical objects the CR mean free path due to the particle scattering by magnetic fluctuations carried by the background plasmas is below the characteristic scale sizes of the plasma flow. In that case the angular distribution of the CRs is nearly isotropic with a small anisotropic part (i.e. both $\beta \ll 1$ and $\chi \ll 1$ in Eq.(18)). Then one can use the diffusion approximation that assumes

$$
f^{c r}(\mathbf{r}, \mathbf{p})=\frac{1}{4 \pi}\left[N^{c r}(\mathbf{r}, p)+\frac{3}{v p} \mathbf{p J}^{c r}(\mathbf{r}, p)\right],
$$

where the diffusive current of CRs is

$$
J_{\alpha}^{c r}=-\kappa_{\alpha \beta} \nabla_{\beta} N^{c r}-\frac{p}{3} \frac{\partial N^{c r}}{\partial p} u_{\alpha}
$$

$\kappa_{\alpha \beta}$ is the momentum-dependent CR diffusion tensor. Then the kinetic equation Eq. (8) reduces to the advection-diffusion equation for the isotropic part of CR distribution $N^{c r}(\mathbf{r}, p, t)$

$$
\frac{\partial N^{c r}}{\partial t}=\nabla_{\alpha} \kappa_{\alpha \beta} \nabla_{\beta} N^{c r}-(\mathbf{u} \nabla) N^{c r}+\frac{p}{3} \frac{\partial N^{c r}}{\partial p} \nabla \mathbf{u}
$$

where $\mathbf{u}(\mathbf{r}, t)$ is the bulk velocity of the background plasma (see, e.g., Toptygin 1983). It is assumed here for simplicity that the scatterers are carried with the plasma bulk velocity, though it is possible to account for the scatterers drift velocity (see, e.g., Skilling 1975). The advantage of this approach is that it is valid for collision operators $I[f]$ more general than just the simple relaxation time approximation given by Eq.(16). In the diffusion approximation the exact form of the collision operator determines the form of the diffusion tensor and its momentum dependence. Therefore, the results obtained within the diffusion approximation are valid for different collision operators.

To explore the effect of CRs on the background plasma one should calculate the first moment of the kinetic equation Eq. (8) for CRs that is the momentum exchange rate between the CRs and the background plasma:

$$
\frac{\partial \mathcal{P}_{\alpha}}{\partial t}+\nabla_{\alpha} P^{c r}+\nabla_{\beta} \Pi_{\alpha \beta}^{\prime}=\left[\frac{1}{c}\left(\mathbf{j}^{c r}-e n_{\mathrm{cr}} \mathbf{u}\right) \times \mathbf{B}+\int \mathbf{p} I[f] d^{3} p\right]_{\alpha},
$$

where $P^{c r}$ is the $\mathrm{CR}$ pressure, the CR momentum density

$$
\mathcal{P}(\mathbf{r}, t)=\int \mathbf{p} f d^{3} p
$$

and the reduced CR momentum flux density $\Pi_{\alpha \beta}^{\prime}$ is defined by

$$
\Pi_{\alpha \beta}^{\prime}=\int p_{\alpha} v_{\beta} f d^{3} p-P^{c r} \delta_{\alpha \beta}
$$


In the diffusion approximation for the steady state (e.g., in the shock rest frame) the first and the third terms in the left hand side of Eq.(45) are small and then Eq.15) can be reduced to

$$
\rho\left(\frac{\partial \mathbf{u}}{\partial t}+(\mathbf{u} \nabla) \mathbf{u}\right)=-\nabla\left(p_{g}+P^{c r}\right)+\frac{1}{4 \pi}(\nabla \times \mathbf{B}) \times \mathbf{B} .
$$

The equation can be applied to longwavelength perturbations. It should be supplied with the continuity equation:

$$
\frac{\partial \rho}{\partial t}+\nabla(\rho \mathbf{u})=0
$$

the energy equations for the background plasma:

$$
\frac{\partial p_{g}}{\partial t}+(\mathbf{u} \nabla) p_{g}+\gamma_{g} p_{g} \nabla \mathbf{u}=0
$$

the MHD induction equation

$$
\frac{\partial \mathbf{B}}{\partial t}=\nabla \times(\mathbf{u} \times \mathbf{B}), \quad \nabla \mathbf{B}=0
$$

and the equation for CR-pressure variations

$$
\frac{\partial P^{c r}}{\partial t}+(\mathbf{u} \nabla) P^{c r}+\gamma_{c r} P^{c r} \nabla \mathbf{u}=\nabla_{\alpha} \bar{\kappa}_{\alpha \beta} \nabla_{\beta} P^{c r}
$$

where $\bar{\kappa}_{\alpha \beta}$ is the CR diffusion tensor averaged over the CR distribution function, $\gamma_{g}$ and $\gamma_{c r}$ - are the adiabatic indexes of the plasma and CRs, respectively.

\section{Acoustic instability driven by the CR pressure gradient}

It was found by Drurv (1984), Dorfi and Drury (1985), Drury and Falle (1986), Drury and Downes (2012) that the force density in Eq.(48) associated with the CR pressure gradient that does not depend on the density of the background plasma results in a specific instability. The effect of magnetic field on the instability was studied by Berezhko (1986) and Chalov (1988b). The analytical study of the instability can be performed for the modes with the wavenumbers below the scale size of the CR pressure gradient $L \sim$ $P^{c r} /\left|\nabla P^{c r}\right|$. In the generic case of the diffusive shock acceleration $L \sim$ $\left(c / u_{\mathrm{s}}\right) \times r_{g} / a$. Following Drury and Falle (1986), Chalov (1988b) for the wavenumber range $k L>1$, but $k r_{g} / a<1$ the mode growth and damping can be derived from the continuity equation for the wave action.

The mode growth rate $\Gamma$ in the simplified geometry where the CR pressure gradient is directed along the unperturbed magnetic field was derived using 
a standard linear analysis of Eqs.(48 - 522) by Chalov (1988a), who obtained the following expression

$$
\begin{aligned}
& \Gamma=\frac{v_{m}^{2}-v_{a}^{2}}{2 v_{m}^{2}-\left(v_{s}^{2}+v_{a}^{2}\right)}\left\{-\frac{\gamma_{c r} P_{0}^{c r}}{\rho_{0}} \frac{k^{2}}{\kappa_{0 \|} k_{\|}^{2}+\kappa_{0 \perp} k_{\perp}^{2}} \frac{v_{m}^{2}-v_{a}^{2} \frac{k^{2}}{k^{2}}}{v_{m}^{2}-v_{a}^{2}} \pm\right. \\
& \left. \pm \frac{\nabla P_{0}^{c r}}{\rho_{0} v_{m}} \frac{k_{\|}}{k}\left[1+\frac{\varsigma \kappa_{0 \|} k^{2}}{\kappa_{0 \|} k_{\|}^{2}+\kappa_{0 \perp} k_{\perp}^{2}} \frac{v_{m}^{2}-v_{a}^{2} \frac{k_{\|}^{2}}{k^{2}}}{v_{m}^{2}-v_{a}^{2}}\right]\right\} .
\end{aligned}
$$

Here $v_{s}$ is the sound speed of the background plasma, $P_{0}^{c r}$ is the unperturbed CR pressure, $\nabla P_{0}^{c r}$ is the gradient of the unperturbed CR pressure, $k_{\|}$and $k_{\perp}$ are the components of the mode wavevector parallel and transverse to the unperturbed magnetic field, respectively, and $\kappa_{0 \|}, \kappa_{0 \perp}$ are the components of the averaged $\mathrm{CR}$ diffusion tensor. It is assumed that the $\mathrm{CR}$ diffusion tensor components scale with the background plasma density as $\bar{\kappa}_{\|, \perp} \sim \rho^{\varsigma}$. The phase velocity of the mode is

$$
v_{m}=\left[v_{s}^{2}+v_{a}^{2} \pm \frac{1}{2} \sqrt{\left(v_{s}^{2}+v_{a}^{2}\right)^{2}-4 v_{s}^{2} v_{a}^{2} \frac{k_{\|}^{2}}{k^{2}}}\right]^{\frac{1}{2}} .
$$

The first term in Eq.(53) is the wave damping rate due to the irreversible stochastic Fermi II CR acceleration effect Achterberg 1979, Bykov and Toptyghin 1979, Ptuskin 1981), while the second and the third terms describe the growth/damping of the modes due to the acoustic instability studied by Drury and Falle (1986). A more general treatment with an arbitrary direction of the unperturbed magnetic field was performed by Chalov (1988b). He accounted for the response of the CR diffusion tensor to both the density and magnetic field variations and found that the latter does not change the character of the angular dependence of the growth rate significantly. A similar angular dependence of the long-wave mode growth rate due to the $\mathrm{CR}$ current driven instability (discussed above in 44.5 ) was found by Bykov et al. (2011c).

In the space plasma with the modest level of the magnetic field fluctuations the local $\mathrm{CR}$ diffusion is anisotropic. For magnetized $\mathrm{CR}$ particles $(a \ll 1)$ the diffusion parallel to the mean magnetic field dominates over the CR diffusion transverse to the mean field, i.e., $\kappa_{0 \|} \gg \kappa_{0 \perp}$. The growth rate of the acoustic instability in the anisotropic system is maximal for the modes propagating nearly transverse to the mean magnetic field $\left(\vartheta \rightarrow \frac{\pi}{2}\right)$. Here $\vartheta$ is the angle between the mode wavevector and the the mean magnetic field. by

The angular dependence of the growth rate Eq.(53) can be approximated

$$
G_{0}(\vartheta)=\frac{\cos \vartheta}{\cos ^{2} \vartheta+\frac{\kappa_{0 \perp}}{\kappa_{0 \|}} \sin ^{2} \vartheta}
$$

where we used $\frac{k_{\|}}{k}=\cos \vartheta, \frac{k_{\perp}}{k}=\sin \vartheta$. The anisotropy of the CR diffusion is determined by the CR particle magnetization (e.g., Toptygin 1983), that is 


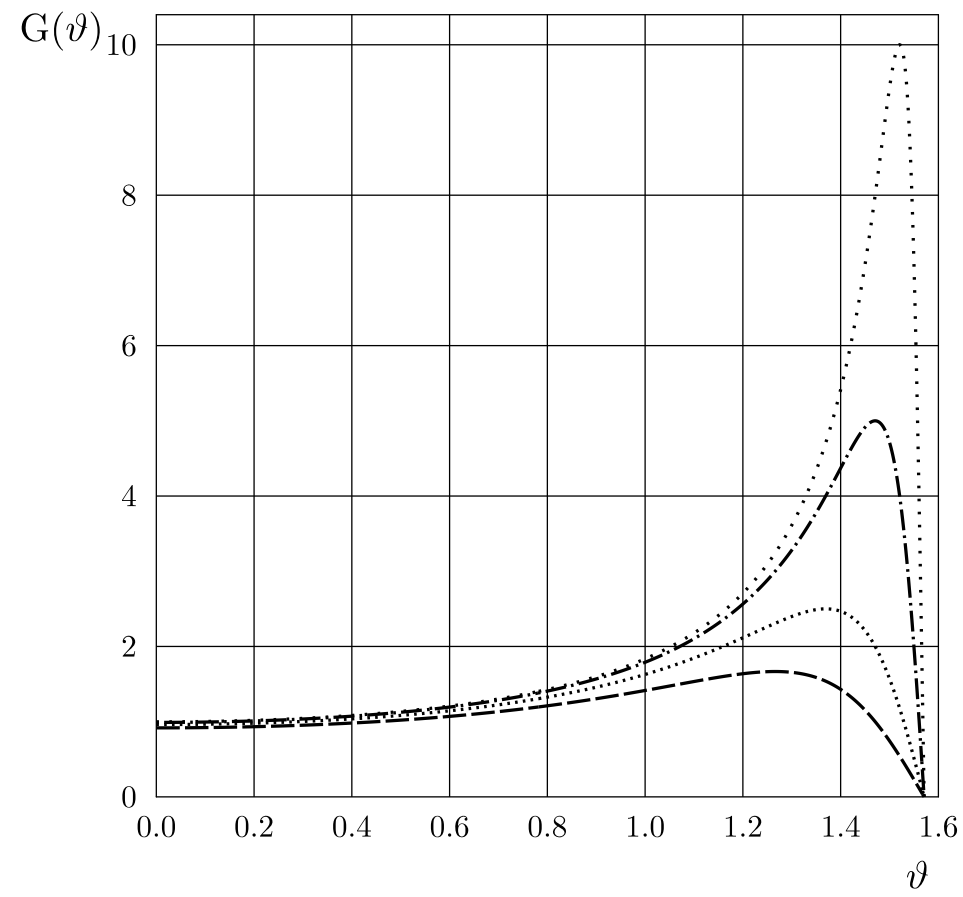

Fig. 8 The characteristic angular dependence of the growth rate of the acoustic instability Eq. (53) for $a=0.3$ (the dashed curve), $a=0.2$ (the dotted curve), $a=0.1$ (the dot-dashed curve), $a=0.05$ (the rare dot curve).

the inverse collisionality parameter $a$, and therefore, $\frac{\kappa_{0 \perp}}{\kappa_{0 \|}} \propto a^{2}$. The maximal growth rate is therefore achieved for the mode propagating at $\cos \vartheta_{\max }=a$, where $G_{\max }\left(\vartheta_{\max }\right)=\frac{1}{2 a}$. The angular dependence of the growth rate of the acoustic instability is illustrated in Figure 8 for various values of collisionality parameter $a$.

The linear perturbation analysis discussed above is based on the diffusion approximation of the CR dynamics in Eqs.(48-52) and, therefore, it is valid for the modes of the wavenumbers above the mean free path of the CRs. A numerical model of the acoustic instability in the nonlinear regime was performed recently by Drury and Downes (2012), who found a significant amplification of magnetic field. The authors assumed a fixed CR diffusion gradient with no response of the CR pressure to the fluctuations, that may affect the model results. 


\section{Self-confinement of CRs near their acceleration sites}

Apart from being a central issue for the acceleration in SNR shocks, the CRdriven instabilities are fast becoming an integral part of CR escape models. One common difficulty with the observational verification of the proton escape is that, in contrast to electrons, they likely remain invisible until they reach some dense material in SNR surroundings. Only there generate they enough $\pi^{0}$ mesons in collisions with other protons and the mesons in turn decay into gamma photons which may be detected. Not surprisingly, the escape of CRs from an SNR is a hot topic of today research in gamma-ray astronomy.

The backbone of the DSA is a self-confinement of accelerated particles by scattering off various magnetic perturbations that particles drive by themselves while streaming ahead of the shock. Most important of them were discussed at some length in this review. Logically, this process should also control the ensuing propagation of CRs, before their density drops below the wave instability threshold. Strictly speaking the CR release (escape) from the accelerator should be treated together with the acceleration, as it does not occur at once for all the particles. But this would be a combination of two difficult enough problems and most of the progress in CR escape was made by considering it separately from acceleration.

Remarkably, even within this limited approach, and under rather loose formulation of the problem, no consensus on the escape mechanism has been reached so far; the dividing lines seem to run across the following issues: (i) does the escape occur isotropically or along the local magnetic field? (ii) does the scattering by the background MHD turbulence control the CR propagation alone or self-excited waves need to be included? (iii) if so, is a quasilinear saturation of self-excited waves sufficient or nonlinear processes of wave damping are crucial to the particle propagation? (iv) if they are, which particular mechanism(s) should be employed?

Starting with (i-ii) we note that most of the early models, and some of the recent ones that target specific remnants, assume isotropic CR propagation from a point source impeded only by the background turbulence (one may call them test particle models, e.g. Aharonian and Atovan 1996, Gabici et al. 2009, Ellison and Bykov 2011, Gabici 2011). It should be noted, however, that e.g., Rosner and Bodo (1996) and Nava and Gabici (2013) adopted a field aligned propagation while Drury (2011) included the finite radius of a SNR shock in the CR escape description. Given the topic of the present short review, however, we focus in this section on models that explicitly include the self-excited waves. Brief reviews of other aspects of CR propagation in the galaxy were given recently by Gabici (2011) and Ptuskin (2012).

The role of self-confinement effects in the CR escape, their subsequent propagation and how these phenomena are treated in different models, can be best demonstrated by writing the following equations that self-consistently describe the CR diffusion and wave generation

$$
\frac{d}{d t} P_{\mathrm{CR}}(p)=\frac{\partial}{\partial z} \frac{\kappa_{\mathrm{B}}}{I} \frac{\partial P_{\mathrm{CR}}}{\partial z}
$$




$$
\frac{d}{d t} I=-v_{\mathrm{a}} \frac{\partial P_{\mathrm{CR}}}{\partial z}-\Gamma I .
$$

Here $v_{a}$ is the Alfvén velocity, $\kappa_{B}$ is the CR diffusion coefficient in Bohm regime, $\kappa_{B}=\mathrm{cr}_{g} / 3$, and the time derivative is taken along the characteristics of unstable Alfvén waves, forward propagating along the field ( $z$ - direction):

$$
\frac{d}{d t}=\frac{\partial}{\partial t}+v_{\mathrm{a}} \frac{\partial}{\partial z}
$$

Eq. (56) above is essentially a well-known convection-diffusion equation, written for the dimensionless $C R$ partial pressure $P_{\mathrm{CR}}$ instead of their distribution function $f(p, t)$. We normalized it to the magnetic energy density $\rho v_{\mathrm{a}}^{2} / 2$ :

$$
P_{\mathrm{CR}}=\frac{4 \pi}{3} \frac{2}{\rho v_{\mathrm{a}}^{2}} v p^{4} f
$$

where $v$ and $p$ are the CR speed and momentum, and $\rho$ - the plasma density. The total CR pressure is normalized to $d \ln p$, similarly to the wave energy density $I$ :

$$
\frac{\left\langle\delta B^{2}\right\rangle}{8 \pi}=\frac{B_{0}^{2}}{8 \pi} \int I(k) d \ln k=\frac{B_{0}^{2}}{8 \pi} \int I(p) d \ln p
$$

Eq.(57) is a wave kinetic equation in which the energy transferred to the waves equals the total work done by the particles, $\left(u+v_{\mathrm{a}}\right) \nabla P_{\mathrm{CR}}$, less the work done on the fluid, $u \nabla P_{\mathrm{CR}}$ (Drury 1983) (we neglect the bulk flow velocity $u$, here and in Eq.(58) assuming that the active phase of acceleration ended by this time). The above interpretation of the wave generation indicates that it operates in a maximum efficiency regime. A formal quasilinear derivation of this equation assumes that the particle momentum $p$ is related to the wave number $k$ by the 'sharpened' resonance condition $k p=e B_{0} / c$ instead of the conventional cyclotron resonance condition $k p_{\|}=e B_{0} / c$ (Skilling 1975), (note that here $k=k_{\|}$). We assume that $\partial P_{\mathrm{CR}} / \partial z \leq 0$ at all times, so that only the forward propagating waves are unstable. The latter inequality is ensured by the formulation of initial value problem symmetric with respect to $z=0$, so we consider the $\mathrm{CR}$ escape into the half-space $z>0$ with the boundary condition $\partial P_{\mathrm{CR}} / \partial z=0$ at $z=0$.

Papers on CR self-confinement discussed below use equations that are largely similar to Eqs.(56][57) but different assumptions are made regarding geometry of particle escape from the source (see (i) above), the character and strength of wave damping $\Gamma$ (iv), and the role of quasilinear wave saturation (iii). Fujita et al. (2011) and Yan et al. (2012) utilize the isotropic escape models (in this case $\partial / \partial z$ should be replaced by $\partial / \partial r$, etc.) while Ptuskin et al. (2008) and Malkov et al. (2012a) assume that particles propagate predominantly along the local large-scale field. Note that Yan et al. (2012) considered the escape from an active accelerator (in Eq. (58), one should include the flow bulk velocity, $v_{a} \rightarrow v_{a}+u$ in this case) and, in addition, they introduce a step-wise increase in CR diffusivity at a certain particle momentum above which particles escape the accelerator. These assumptions 


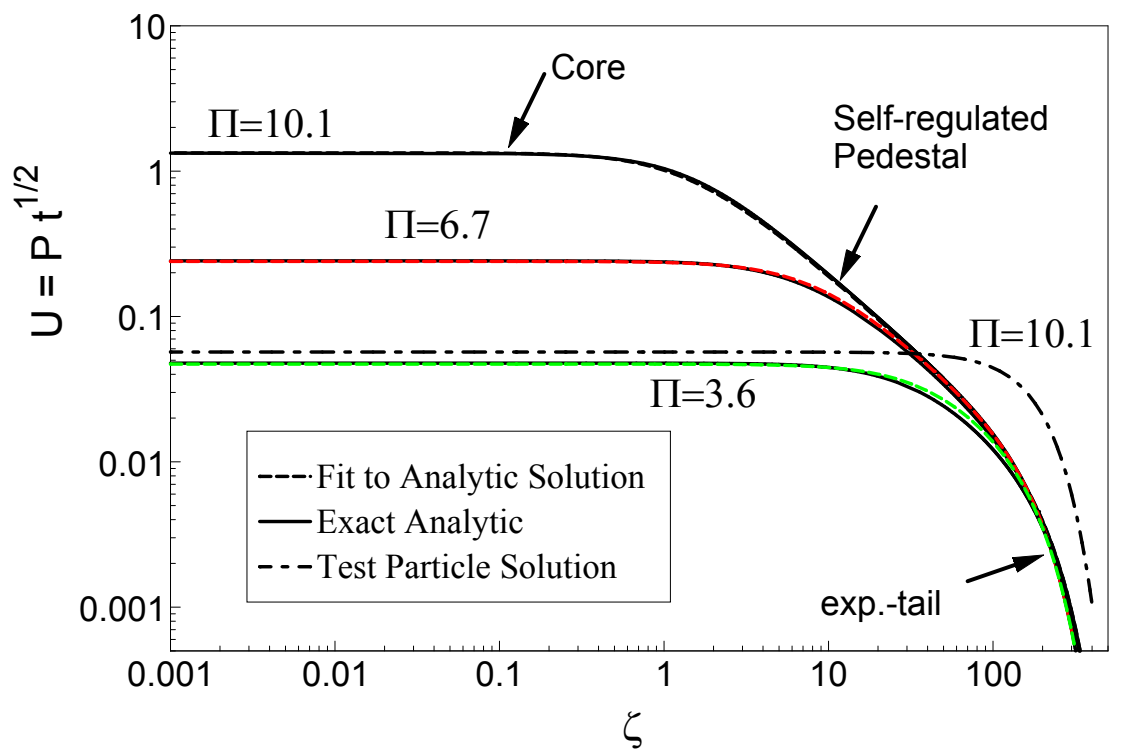

Fig. 9 Spatial distribution of CR partial pressure (as a function of $\zeta=z / \sqrt{a v_{a} t}$, multiplied by $v_{a}^{3 / 2} \sqrt{a t} / \kappa_{B}$ ) shown for integrated values of this quantity $\Pi=3.6$; $6.7 ; 10.1$ and for for the background diffusivity $D_{I S M}=10^{4}$. Exact analytic solutions are shown with the solid lines while the interpolations given by Equation (65) are shown with the dashed lines. For comparison, a formal test particle solution for $\Pi=10.1$ is also shown with the dot-dashed line. Note the three characteristic zones of the CR confinement: the innermost flat top core, the scale invariant $(1 / \zeta)$ pedestal, and the exponential decay zone.

make it difficult to compare their results with those of the remaining three papers. In these, Fujita et al. (2011) presented the results of numerical integration of Eqs.5657) (in a spherical symmetry) with neglected damping term $\Gamma$. The results indicate a considerable delay of diffusion from the source due to a self-confinement.

However, in the regions where magnetic perturbations are weak, i.e. $I \ll$ 1, the field aligned CR transport is appropriate, as the perpendicular diffusion is suppressed, $\kappa_{\perp} \simeq I^{2} \kappa_{\|} \ll \kappa_{\|} \simeq \kappa_{B} / I$. Taking into account the condition $I_{\text {ISM }} \ll 1$, such regime appears inevitable far away from the source and at late times when particles are spread over a large volume and the waves are driven only weakly. At earlier times and close to the region of the initial localization of CRs, an estimate $\kappa_{\perp} \sim \kappa_{\|} \sim \kappa_{\mathrm{B}}$ appears to be adequate. 
Both analytical models by Ptuskin et al. (2008) and Malkov et al. (2012a), however, do not embrace the general case and rely on the assumption $\kappa_{\perp} \ll$ $\kappa_{\|}$thus considering a field-aligned escape. At the same time, they are different in further simplifications made, that lead to rather different results, both quantitatively and qualitatively.

Ptuskin et al. (2008) neglect $d I / d t$ on the l.h.s of Eq.(57) thus balancing the driving term with the damping term on its r.h.s and assume a Kolmogorov dissipation for $\Gamma$,

$$
\Gamma=k v_{a} \sqrt{I} /\left(2 C_{K}\right)^{3 / 2}
$$

with $C_{K} \approx 3.6$ and $k \simeq 1 / r_{g}(p)$ being the resonant wave number. Therefore, only one equation (56) needs to be solved which lead to the following selfsimilar solution (in notations and normalization used in Eqs.(56]577)

$$
P_{C R}=\frac{4 \cdot 3^{-3 / 2}}{t^{\prime 3 / 2} \sqrt{\sigma+(k z)^{4} / t^{\prime 6}}}
$$

where the dimensionless time $t^{\prime}=\left(\kappa_{B} k^{2} / 2 C_{K}\right) t, \sigma=\Gamma^{8}(1 / 4) / \pi^{2} 3^{6} \eta^{4}$, and $\Gamma$ is the gamma function. The single important parameter this solution depends on is the integrated (along the field line) CR partial pressure

$$
\eta=2 k \int_{0}^{\infty} P_{C R} d z
$$

Therefore, the CR density decays at the source as $\propto t^{-3 / 2}$ and the flattopped, self-confined part of the CR distribution spreads as $z \propto t^{3 / 2}$, both pointing at the superdiffusive CR transport. The reason is clearly in a very strong wave damping due to the Kolmogorov dissipation. For the same reason this solution does not recover the test particle asymptotic result $P_{C R} \propto$ $t^{-1 / 2} \exp \left(-z^{2} / 4 D_{\text {ISM }} t\right)$, physically expected in $z, t \rightarrow \infty$ limit in the interstellar medium with the background diffusion coefficient $D_{\text {ISM }}$.

An alternative choice of damping mechanism is the Goldreich and Sridhar (1997) MHD spectrum, which seems to be more appropriate in $I \lesssim 1$ regime under not too strong MHD cascade (Farmer and Goldreich 2004, Beresnyak and Lazarian 2008, Yan et al. 2012). The damping rate in this case is

$$
\Gamma=v_{a} \sqrt{\frac{k}{L}}
$$

where $L$ is the outer scale of turbulence which may be as large as $100 p c$. Not only is this damping orders of magnitude (roughly a factor $\sqrt{r_{g} / L}$ ) lower than the Kolmogorov one but, as it does not depend on $I$ and can be considered as coordinate independent, it allows for the following ('quasilinear') integral of the system of Eqs.(56) and (57):

$$
P_{\mathrm{CR}}(z, t)=P_{\mathrm{CR} 0}\left(z^{\prime}\right)-\frac{\kappa_{\mathrm{B}}}{v_{\mathrm{a}}} \frac{\partial}{\partial z} \ln \frac{I(z, t)}{I_{0}\left(z^{\prime}\right)}
$$


Here $P_{\mathrm{CR} 0}(z)$ and $I_{0}(z)$ are the initial distributions of the CR partial pressure and the wave energy density, respectively, and $z^{\prime}=z-v_{\mathrm{a}} t$. Substituting $P_{C R}$ in Eq. (57) and neglecting slow convection with $v_{a}$ in Eq.(58), we arrive at the following diffusion equation for $I$

$$
\frac{\partial I}{\partial t}=\frac{\partial}{\partial z} \frac{\kappa_{B}}{I} \frac{\partial I}{\partial z}-\Gamma I-v_{a} \frac{\partial P_{C R 0}}{\partial z}
$$

The equation is supplemented with the boundary condition $I \rightarrow I_{I S M}$, for $|z| \rightarrow \infty$. Outside of the region where $P_{C R} \neq 0$, the last term on the r.h.s. may be neglected. The second term may be eliminated by replacing $I \exp (\Gamma t) \rightarrow$ $I, \int_{0}^{t} \exp (\Gamma t) d t \rightarrow t$. However, if $\Gamma$ is taken in the form of Eq.(63), it is fairly small due to the factor $\sqrt{r_{g} / L} \ll 1$. We may simply neglect it. The solution for $I$ and $P_{C R}(z, t)$ may be found in an implicit form (see Malkov et al. 2012a for details). However, there exists a very accurate convenient interpolation formula that can be represented as follows

$$
P_{\mathrm{CR}}=\frac{2 \kappa_{\mathrm{B}}(p)}{v_{\mathrm{a}}^{3 / 2} \sqrt{L_{c} t}}\left[\zeta^{5 / 3}+\left(D_{\mathrm{NL}}\right)^{5 / 6}\right]^{-3 / 5} e^{-\zeta^{2} / 4 D_{\mathrm{ISM}}}
$$

where $L_{c}$ is the size of the initial CR cloud, $\zeta=z / \sqrt{v_{a} L_{c} t}$, and $D_{\mathrm{NL}}=$ $C(\Pi) D_{\text {ISM }} \exp (-\Pi)$, with $\Pi$ being a normalized integrated pressure

$$
\Pi=\frac{v_{a}}{\kappa_{B}} \int_{0}^{\infty} P_{C R} d z
$$

and $D_{\text {ISM }}$ is the normalized background diffusivity

$$
D_{\mathrm{ISM}}=\frac{\kappa_{B}}{v_{a} L_{c}} I_{\mathrm{ISM}}^{-1}
$$

while $C \sim 1$, for $\Pi \gg 1$ and $C \sim \Pi^{-2}$, for $\Pi \ll 1$.

The representation of the solution given in Eq. (65) is convenient in that the function $\sqrt{t} P_{C R}(\zeta)$ does not depend on $t$, so that the solution can be shown for all $t, z$ with only one curve, Figure 9. To summarize these results, the self-regulated normalized $\left(\mathcal{P}_{C R}=v_{\mathrm{a}} L_{c} P_{\mathrm{CR}} / \kappa_{\mathrm{B}}(p)\right) \mathrm{CR}$ partial pressure profile $\mathcal{P}_{C R}$ comprises the following three zones $(\Pi \gg 1)$ : (i) a quasi-plateau (core) at small $z / \sqrt{t}<\sqrt{D_{\mathrm{NL}}}$ of a height $\sim\left(D_{\mathrm{NL}} t\right)^{-1 / 2}$, which is elevated by a factor $\sim \Pi^{-1} \exp (\Pi / 2) \gg 1$, compared to the test particle solution because of the strong quasi-linear suppression of the CR diffusion coefficient with respect to its background (test particle) value $D_{\mathrm{ISM}}: D_{\mathrm{NL}} \sim D_{\mathrm{ISM}} \exp (-\Pi)$ (ii) next to the core, where $\sqrt{D_{\mathrm{NL}}}<z / \sqrt{t}<\sqrt{D_{\mathrm{ISM}}}$, the profile is scale invariant, $\mathcal{P}_{C R} \approx 2 / z$. The $\mathrm{CR}$ distribution in this "pedestal" region is fully self-regulated, independent of $\Pi$ and $D_{\text {ISM }}$ for $\Pi \gg 1$, (iii) the tail of the distribution at $z / \sqrt{t}>\sqrt{D_{\text {ISM }}}$ is similar in shape to the test particle solution in $1 \mathrm{D}$ but it saturates with $\Pi \gg 1$, so that the CR partial pressure is $\propto$ $\left(D_{\text {ISM }} t\right)^{-1 / 2} \exp \left(-z^{2} / 4 D_{\text {ISM }} t\right)$, independent of the strength of the CR source $\Pi$, in contrast to the test-particle result which scales as $\propto \Pi$. Because of the 
CR diffusivity reduction, the CR cloud half-life is increased and the cloud width is decreased, compared to the test particle solution.

Depending on the functions $\Pi(p)$ and $D_{\text {ISM }}(p)$, the resulting CR spectrum generally develops a spectral break for the fixed values of $z$ and $t$ such that $z^{2} / t \sim D_{\mathrm{NL}}(p) \sim D_{\mathrm{ISM}} \exp (-\Pi)$.

\section{Summary}

Cosmic rays, being a highly non-equilibrium component, often comprise an energy density that is comparable to the ram pressure of energetic plasma flows and magnetic fields in astrophysical sources with high energy release such as supernova remnants, fast stellar winds, and astrophysical jets of different scales. CRs may also play a role in the global dynamics of interstellar gas in galaxies, in particular, they may support galactic winds. In the presence of gravitation, the buoyancy of CRs and magnetic field at galactic scales may result in the magnetic Parker instability (Parker 1966, 1967, Shu 1974, Ryu et al. 2003, Hanasz et al. 2009). The local CR diffusion is an important factor for the Parker instability to occur.

The microphysical instabilities discussed above lay the groundwork for detailed simulations of the global interstellar matter dynamics. In this review we addressed the recent progress in understanding of the CR-driven instabilities with special attention to non-relativistic shocks. We started with a quasilinear analysis of the growth rates of the instabilities driven by anisotropic and inhomogeneous CR distributions. Time dependent nonlinear simulations are needed to draw conclusions about the saturation level and the spectra of magnetic fluctuations produced by the non-equilibrium CR distributions. We used numerical simulations to illustrate the nonlinear dynamics of magnetic fluctuations. The CR-driven instabilities are shown to be crucial for modeling particle acceleration sources and the CR escape from the sources into the interstellar matter.

Acknowledgements We thank Andre Balogh and the ISSI staff for providing an inspiring atmosphere at the International Space Science Institute Workshop in Bern in 2012, which has led to new collaborations and scientific progress. Computing resources to A.B. were provided by the Swedish National Allocations Committee at the Center for Parallel Computers at the Royal Institute of Technology in Stockholm and the High Performance Computing Center North in Umeå. A.B. was supported in part by the European Research Council under the AstroDyn Research Project No. 227952 and the Swedish Research Council under the project grant 621 2011-5076. A.M.B. and S.M.O. acknowledge support from the RAS Programs P21 and OFN 16, and from the Ministry of Education and Science of Russian Federation (Agreement No. 8409, 2012). Some of the simulations were performed at the Joint Supercomputing Centre (JSCC RAS) and the Supercomputing Centre at Ioffe Institute, St.Petersburg. M.M. acknowledges support by the Department of Energy, Grant No. DE-FG02-04ER54738.

\section{References}

Achterberg A (1979) The energy spectrum of electrons accelerated by weak magnetohydrodynamic turbulence. A\&A 76:276-286 
Achterberg A (1983) Modification of scattering waves and its importance for shock acceleration. A\&A 119:274-278

Achterberg A, Blandford RD (1986) Transmission and damping of hydromagnetic waves behind a strong shock front - Implications for cosmic ray acceleration. MNRAS 218:551-575

Aharonian FA, Atoyan AM (1996) On the emissivity of $\pi^{\wedge} 0^{\wedge}$-decay gamma radiation in the vicinity of accelerators of galactic cosmic rays. A\&A 309:917-928

Amato E (2011) The streaming instability: a review . Mem. Soc. Astron. Italiana $82: 806$

Amato E, Blasi P (2009) A kinetic approach to cosmic-ray-induced streaming instability at supernova shocks. MNRAS 392:1591-1600, 0806.1223

Bell AR (1978) The acceleration of cosmic rays in shock fronts. I. MNRAS 182:147156

Bell AR (2004) Turbulent amplification of magnetic field and diffusive shock acceleration of cosmic rays. MNRAS 353:550-558

Bell AR (2005) The interaction of cosmic rays and magnetized plasma. MNRAS 358:181-187

Bell AR, Lucek SG (2001) Cosmic ray acceleration to very high energy through the non-linear amplification by cosmic rays of the seed magnetic field. MNRAS 321:433-438

Beresnyak A, Jones TW, Lazarian A (2009) Turbulence-Induced Magnetic Fields and Structure of Cosmic Ray Modified Shocks. ApJ 707:1541-1549, 0908.2806

Beresnyak A, Lazarian A (2008) Wave Decay in Magnetohydrodynamic Turbulence. ApJ 678:961-967, 0805.0630

Berezhko EG (1986) Instability in a Shock Propagating Through Gas with a Cosmic-Ray Component. Soviet Astronomy Letters 12:352-+

Berezhko EG, Krymskii GF (1988) REVIEWS OF TOPICAL PROBLEMS: Acceleration of cosmic rays by shock waves. Soviet Physics Uspekhi 31:27-51

Berezinskii VS, Bulanov SV, Dogiel VA, Ginzburg VL, et al. (1990) Astrophysics of cosmic rays. Amsterdam: North-Holland, 1990

Blackman EG, Field GB (2002) New Dynamical Mean-Field Dynamo Theory and Closure Approach. Physical Review Letters 89(26):265 007-+, arXiv: astro-ph/0207435

Blandtord R, Eichler D (1987) Particle acceleration at astrophysical shocks: A theory of cosmic ray origin. Phys. Reports 154:1-75

Brandenburg A (2005) Turbulence and its parameterization in accretion discs. Astronomische Nachrichten 326:787-797, arXiv:astro-ph/0510015

Brandenburg A (2009a) Advances in Theory and Simulations of Large-Scale Dynamos. Space Science Reviews 144:87-104, 0901.0329

Brandenburg A (2009b) Large-scale Dynamos at Low Magnetic Prandtl Numbers. ApJ 697:1206-1213, 0808.0961

Brandenburg A, Rädler KH, Kemel K (2012) Mean-field transport in stratified and/or rotating turbulence. A\&A 539:A35, 1108.2264

Bykov AM (1982) Interstellar Turbulence and Shock Waves. Soviet Astronomy Letters 8:320

Bykov AM (1988) A Model for the Generation of Interstellar Turbulence. Soviet Astronomy Letters 14:60

Bykov AM, Ellison DC, Osipov SM, Pavlov GG, et al. (2011a) X-ray Stripes in Tycho's Supernova Remnant: Synchrotron Footprints of a Nonlinear Cosmicray-driven Instability. ApJ 735:L40, 1106.3441

Bykov AM, Ellison DC, Renaud M (2012) Magnetic Fields in Cosmic Particle Acceleration Sources. Space Sci. Rev. 166:71-95, 1105.0130

Bykov AM, Gladilin PE, Osipov SM (2011b) Particle acceleration at supernova shocks in young stellar clusters. Mem. Soc. Astron. Italiana 82:800, 1111.2587

Bykov AM, Osipov SM, Ellison DC (2011c) Cosmic ray current driven turbulence in shocks with efficient particle acceleration: the oblique, long-wavelength mode instability. MNRAS 410:39-52, 1010.0408

Bykov AM, Toptyghin IN (1979) Particle Acceleration by Astrophysical Supersonic Turbulence. In: 16th International Cosmic Ray Conference, Kyoto, Japan, 
vol. 2 , p. 66

Bykov AM, Uvarov YA, Ellison DC (2008) Dots, Clumps, and Filaments: The Intermittent Images of Synchrotron Emission in Random Magnetic Fields of Young Supernova Remnants. ApJ 689:L133-L136, 0811.2498

Caprioli D, Blasi P, Amato E, Vietri M (2008) Dynamical Effects of SelfGenerated Magnetic Fields in Cosmic-Ray-modified Shocks. ApJ 679:L139L142, arXiv:0804.2884

Cassam-Chenaï G, Hughes JP, Ballet J, Decourchelle A (2007) The Blast Wave of Tycho's Supernova Remnant. ApJ 665:315-340, arXiv:astro-ph/0703239

Chalov SV (1988a) Diffusive Shock Instability in Plasma Modified by Cosmic-Rays. Soviet Astronomy Letters 14:114-+

Chalov SV (1988b) Instability of the structure of strong oblique MHD cosmic-ray shocks. Astroph. Space Sci. 148:175-187

Diamond PH, Malkov MA (2007) Dynamics of Mesoscale Magnetic Field in Diffusive Shock Acceleration. ApJ 654:252-266, arXiv:astro-ph/0605374

Dorfi EA, Drury LO (1985) A cosmic ray driven instability. In: International Cosmic Ray Conference (ed. Jones FC), vol. 3 of International Cosmic Ray Conference, pp. $121-123$

Drury LO (1983) An introduction to the theory of diffusive shock acceleration of energetic particles in tenuous plasmas. Reports on Progress in Physics 46:9731027

Drury LO (1984) Reaction effects in diffusive shock acceleration. Advances in Space Research 4:185-191

Drury LO (2011) Escaping the accelerator: how, when and in what numbers do cosmic rays get out of supernova remnants? MNRAS 415:1807-1814, 1009.4799

Drury LO, Downes TP (2012) Turbulent magnetic field amplification driven by cosmic ray pressure gradients. MNRAS 427:2308-2313, 1205.6823

Drury LO, Falle SAEG (1986) On the Stability of Shocks Modified by Particle Acceleration. MNRAS 223:353

Drury LO, Markiewicz WJ, Voelk HJ (1989) Simplified models for the evolution of supernova remnants including particle acceleration. A\&A 225:179-191

Durrer R, Caprini C (2003) Primordial magnetic fields and causality. Journal of Cosmology and Astroparticle Physics11:010, arXiv:astro-ph/0305059

Ellison DC, Bykov AM (2011) Gamma-ray Emission of Accelerated Particles Escaping a Supernova Remnant in a Molecular Cloud. ApJ 731:87, 1102.3885

Eriksen KA, Hughes JP, Badenes C, Fesen R, et al. (2011) Evidence for Particle Acceleration to the Knee of the Cosmic Ray Spectrum in Tycho's Supernova Remnant. Astrophysical Journal 728:L28+, 1101.1454

Farmer AJ, Goldreich P (2004) Wave Damping by Magnetohydrodynamic Turbulence and Its Effect on Cosmic-Ray Propagation in the Interstellar Medium. ApJ 604:671-674, arXiv:astro-ph/0311400

Fraschetti F (2013) Turbulent amplification of magnetic field driven by dynamo effect at rippled shocks. ArXiv e-prints 1304.4956

Fujita Y, Takahara F, Ohira Y, Iwasaki K (2011) Alfvén wave amplification and self-containment of cosmic rays escaping from a supernova remnant. MNRAS 415:3434-3438, 1105.0683

Gabici S (2011) Cosmic ray escape from supernova remnants . Mem. Soc. Astron. Italiana $82: 760,1108.4844$

Gabici S, Aharonian FA, Casanova S (2009) Broad-band non-thermal emission from molecular clouds illuminated by cosmic rays from nearby supernova remnants. MNRAS 396:1629-1639, 0901.4549

Giacalone J, Jokipii JR (2007) Magnetic Field Amplification by Shocks in Turbulent Fluids. ApJ 663:L41-L44

Goldreich P, Sridhar S (1997) Magnetohydrodynamic Turbulence Revisited. ApJ 485:680, arXiv: astro-ph/9612243

Hanasz M, Otmianowska-Mazur K, Kowal G, Lesch H (2009) Cosmic-ray-driven dynamo in galactic disks. A parameter study. A\&A 498:335-346, 0812.3906

Helder EA, Vink J, Bykov AM, Ohira Y, et al. (2012) Observational Signatures of Particle Acceleration in Supernova Remnants. Space Sci. Rev. 173:369-431, 
1206.1593

Jones FC, Ellison DC (1991) The plasma physics of shock acceleration. Space Science Reviews 58:259-346

Kevlahan NKR (1997) The vorticity jump across a shock in a non-uniform flow. Journal of Fluid Mechanics 341:371-384

Krymskii GF (1977) A regular mechanism for the acceleration of charged particles on the front of a shock wave. Akademiia Nauk SSSR Doklady 234:1306-1308

Kulsrud RM, Cen R, Ostriker JP, Ryu D (1997) The Protogalactic Origin for Cosmic Magnetic Fields. ApJ 480:481, arXiv:astro-ph/9607141

Malkov MA, Diamond PH (2006) Nonlinear Shock Acceleration beyond the Bohm Limit. ApJ 642:244-259, arXiv:astro-ph/0509235

Malkov MA, Diamond PH (2009) Nonlinear Dynamics of Acoustic Instability in a Cosmic Ray Shock Precursor and its Impact on Particle Acceleration. ApJ 692:1571-1581

Malkov MA, Diamond PH, Sagdeev RZ, Aharonian FA, et al. (2012a) Analytic Solution for Self-regulated Collective Escape of Cosmic Rays from their Acceleration Sites. submitted to ApJ 1207.4728

Malkov MA, Drury LO (2001) Nonlinear theory of diffusive acceleration of particles by shock waves. Reports on Progress in Physics 64:429-481

Malkov MA, Sagdeev RZ, Diamond PH (2012b) Magnetic and Density Spikes in Cosmic-Ray Shock Precursors. ApJ 748:L32, 1110.0257

Marcowith A, Lemoine M, Pelletier G (2006) Turbulence and particle acceleration in collisionless supernovae remnant shocks. II. Cosmic-ray transport. A\&A 453:193-202, arXiv:astro-ph/0603462

McKenzie JF, Voelk HJ (1982) Non-linear theory of cosmic ray shocks including self-generated Alfven waves. A\&A 116:191-200

McKenzie JF, Westphal KO (1970) Interaction of Hydromagnetic Waves with Hydromagnetic Shocks. Physics of Fluids 13:630-640

Nava L, Gabici S (2013) Anisotropic cosmic ray diffusion and gamma-ray production close to supernova remnants, with an application to W28. MNRAS 429:1643-1651, 1211.1668

Noerdlinger PD, Yui AKM (1968) Persistence of the Firehouse Instability in Highly Relativistic Plasmas. ApJ 151:901

Ohira Y, Murase K, Yamazaki R (2011) Gamma-rays from molecular clouds illuminated by cosmic rays escaping from interacting supernova remnants. MNRAS 410:1577-1582, 1007.4869

Parker EN (1966) The Dynamical State of the Interstellar Gas and Field. ApJ $145: 811$

Parker EN (1967) The Dynamical State of the Interstellar Gas and Field. III. Turbulence and Enhanced Diffusion. ApJ 149:535

Pelletier G, Lemoine M, Marcowith A (2006) Turbulence and particle acceleration in collisionless supernovae remnant shocks. I. Anisotropic spectra solutions. A\&A 453:181-191, arXiv:astro-ph/0603461

Ptuskin V (2012) Propagation of galactic cosmic rays. Astroparticle Physics 39:4451

Ptuskin VS (1981) Influence of cosmic rays on propagation of long magneto hydrodynamic waves. Astroph. Space Sci. 76:265-278

Ptuskin VS, Zirakashvili VN, Plesser AA (2008) Non-linear diffusion of cosmic rays. Advances in Space Research 42:486-490

Reville B, O'Sullivan S, Duffy P, Kirk JG (2008) The transport of cosmic rays in self-excited magnetic turbulence. MNRAS 386:509-515, 0802.0109

Reynolds SP (2008) Supernova Remnants at High Energy. ARA\&A 46:89-126

Riquelme MA, Spitkovsky A (2009) Nonlinear Study of Bell's Cosmic Ray CurrentDriven Instability. ApJ 694:626-642, 0810.4565

Riquelme MA, Spitkovsky A (2010) Magnetic Amplification by Magnetized Cosmic Rays in Supernova Remnant Shocks. ApJ 717:1054-1066, 0912.4990

Rogachevskii I, Kleeorin N, Brandenburg A, Eichler D (2012) Cosmic-Ray Currentdriven Turbulence and Mean-field Dynamo Effect. ApJ 753:6, 1204.4246

Rosner R, Bodo G (1996) The Origin of Filaments in the Interstellar Medium. ApJ 
470:L49

Ryu D, Kang H, Jones TW (1993) The stability of cosmic-ray-dominated shocks A secondary instability. ApJ 405:199-206

Ryu D, Kim J, Hong SS, Jones TW (2003) The Effect of Cosmic-Ray Diffusion on the Parker Instability. ApJ 589:338-346, arXiv:astro-ph/0301625

Sagdeev RZ, Shafranov VD (1961) On the instability of a plasma with anisotropic distribution of velocities in magnetic field. Soviet Phys JETP 12(1):130-132

Schlickeiser R (2002) Cosmic Ray Astrophysics. Springer, Berlin

Schrinner M, Rädler KH, Schmitt D, Rheinhardt M, et al. (2005) Mean-field view on rotating magnetoconvection and a geodynamo model. Astronomische Nachrichten 326:245-249

Schure KM, Bell AR (2011) A long-wavelength instability involving the stress tensor. MNRAS 418:782-788, 1107.5817

Schure KM, Bell AR, O'C Drury L, Bykov AM (2012) Diffusive Shock Acceleration and Magnetic Field Amplification. Space Sci. Rev. 173:491-519, 1203.1637

Shapiro VD, Quest KB, Okolicsanyi M (1998) Non-resonant firehose instability: Consequences for the theory of cosmic ray acceleration. Geophysical Research Letters 25:845-848

Shu FH (1974) The Parker Instability in Differentially-rotating Disks. A\&A 33:55

Skilling J (1975) Cosmic ray streaming. I - Effect of Alfven waves on particles. MNRAS 172:557-566

Toptygin IN (1983) Cosmic rays in interplanetary magnetic fields

Treumann RA, Baumjohann W (1997) Advanced space plasma physics

Uchiyama Y, Aharonian FA, Tanaka T, et al (2007) Extremely fast acceleration of cosmic rays in a supernova remnant. Nat 449:576-578

Vink J (2012) Supernova remnants: the X-ray perspective. Astron. Astroph. Reviews 20:49, 1112.0576

Vladimirov AE, Bykov AM, Ellison DC (2009) Spectra of Magnetic Fluctuations and Relativistic Particles Produced by a Nonresonant Wave Instability in Supernova Remnant Shocks. ApJ 703:L29-L32, 0908.2602

Yan H, Lazarian A, Schlickeiser R (2012) Cosmic-Ray Streaming from Supernova Remnants and Gamma-Ray Emission from nearby Molecular Clouds. ApJ $745: 140,1111.2410$

Zirakashvili VN, Ptuskin VS (2008) Diffusive Shock Acceleration with Magnetic Amplification by Nonresonant Streaming Instability in Supernova Remnants. ApJ 678:939-949, 0801.4488

Zirakashvili VN, Ptuskin VS, Völk HJ (2008) Modeling Bell's Nonresonant CosmicRay Instability. ApJ 678:255-261, 0801.4486

Zweibel EG (1979) Energetic particle trapping by Alfven wave instabilities. In: Particle Acceleration Mechanisms in Astrophysics (ed. J Arons, C McKee, \& C Max), vol. 56 of American Institute of Physics Conference Series, pp. 319-328

Zweibel EG (2003) Cosmic-Ray History and Its Implications for Galactic Magnetic Fields. ApJ 587:625-637, arXiv:astro-ph/0212559

Zweibel EG, Everett JE (2010) Environments for Magnetic Field Amplification by Cosmic Rays. ApJ 709:1412-1419, 0912.3511

\section{Appendix A}

The dispersion equation Eq. (20) can be expressed in the elementary functions by evaluating Eq. (22) and Eq.(23) as

$$
\begin{gathered}
\sigma_{0}(p)=\frac{3}{2 x^{2}}+\frac{3}{8 x}\left(1-\frac{1}{x^{2}}+\left(\frac{a}{x}\right)^{2}\right) \Psi_{1}-\frac{3 a}{2 x^{3}} \Psi_{2} \mp \\
\mp i\left\{\frac{3}{4 x}\left(1-\frac{1}{x^{2}}+\left(\frac{a}{x}\right)^{2}\right) \Psi_{2}-\frac{3 a}{2 x^{2}}+\frac{3 a}{4 x^{3}} \Psi_{1}\right\},
\end{gathered}
$$




$$
\begin{gathered}
\sigma_{1}(p)=\mp \frac{1}{x} \pm \frac{3}{2 x^{3}} \mp \frac{3}{2 x}\left(\frac{a}{x}\right)^{2} \pm \frac{3}{8 x^{2}}\left(1-\frac{1}{x^{2}}+3\left(\frac{a}{x}\right)^{2}\right) \Psi_{1} \pm \\
\pm \frac{3 a}{4 x^{2}}\left(1-\frac{3}{x^{2}}+\left(\frac{a}{x}\right)^{2}\right) \Psi_{2}- \\
-i\left\{\frac{3}{4 x^{2}}\left(1-\frac{1}{x^{2}}+3\left(\frac{a}{x}\right)^{2}\right) \Psi_{2}-\frac{3 a}{x^{3}}-\frac{3 a}{8 x^{2}}\left(1+\left(\frac{a}{x}\right)^{2}-\frac{3}{x^{2}}\right) \Psi_{1}\right\}(67) \\
\Psi_{1}(x)=\ln \left[\frac{(x+1)^{2}+a^{2}}{(x-1)^{2}+a^{2}}\right] \\
\Psi_{2}(x)=\operatorname{arctg}\left(\frac{x+1}{a}\right)+\operatorname{arctg}\left(\frac{x-1}{a}\right)
\end{gathered}
$$

